Review

\title{
A Systematic Review of Location Aware Schemes in the Internet of Things
}

\author{
Muneeb A. Khan ${ }^{1}\left(\mathbb{D}\right.$, Abdul Saboor ${ }^{2}{ }^{(0)}$, Hyun-chul Kim ${ }^{1}$ and Heemin Park ${ }^{1, *(1)}$ \\ 1 Department of Software, Sangmyung University, Cheonan 31066, Korea; \\ muneebkhan046@gmail.com (M.A.K.); hkim@smu.ac.kr (H.-c.K.) \\ 2 Department of Electrical Engineering (ESAT), KU Leuven, 3000 Leuven, Belgium; asaboor@esat.kuleuven.be \\ * Correspondence: heemin@smu.ac.kr
}

check for updates

Citation: Khan, M.A.; Saboor, A.; Kim, H.-c.; Park, H. A Systematic Review of Location Aware Schemes in the Internet of Things. Sensors 2021, 21, 3228. https://doi.org/ $10.3390 / \mathrm{s} 21093228$

Academic Editor: Antonio Guerrieri

Received: 30 March 2021

Accepted: 27 April 2021

Published: 6 May 2021

Publisher's Note: MDPI stays neutral with regard to jurisdictional claims in published maps and institutional affiliations.

Copyright: (c) 2021 by the authors. Licensee MDPI, Basel, Switzerland. This article is an open access article distributed under the terms and conditions of the Creative Commons Attribution (CC BY) license (https:// creativecommons.org/licenses/by/ $4.0 /)$.

\begin{abstract}
The rapid development in wireless technologies is positioning the Internet of Things (IoT) as an essential part of our daily lives. Localization is one of the most attractive applications related to IoT. In the past few years, localization has been gaining attention because of its applicability in safety, health monitoring, environment monitoring, and security. As a result, various localization-based wireless frameworks are being presented to improve such applications' performances based on specific key performance indicators (KPIs). Therefore, this paper explores the recently proposed localization schemes in IoT. Initially, this paper explains the major KPIs of localization. After that, a thorough comparison of recently proposed localization schemes based on the KPIs is presented. The comparison includes an overview, architecture, network structure, performance parameters, and target KPIs. At the end, possible future directions are presented for the researchers working in this domain.
\end{abstract}

Keywords: Internet of Things (IoT); Location of Things; target localization; wireless sensor network (WSN); review

\section{Introduction}

The Internet is a necessity for billions of people worldwide who need it to complete their daily tasks [1,2]. Furthermore, it provides various entertainment applications such as movies, music, and gaming. One estimate states that more than $58 \%$ of the world's population has access to the Internet to perform such daily tasks. The popularity and growth of the Internet exponentially increased (roughly 1170\%) from 2000 to 2020 [3]. It is transforming the world into a global village where people can connect and communicate worldwide using the Internet.

The Internet allows different devices and appliances to connect and communicate, which led to a new domain called the Internet of Things (IoT) [4-8]. The IoT architecture consists of three layers: the physical layer, the network layer, and the application layer, as shown in Figure 1. The physical layer consists of various sensors attached to the subject. These sensors collect the data/information from the subject. Generally, the nature of data depends on the IoT application requirements. For example, localization-based applications require monitoring and collection of the locality information of the subject. Likewise, telehealth applications need to monitor the vitals of patients, and agricultural applications measure the temperature. The physical layer forwards the data to the network layer.

The network layer is the middle layer between the application and sensor layer in IoT architecture. The network layer aims to transmit the data/information from the sensors to the application layer. The medium of data transmission (wireless or wired) varies based on the application and requirements. Furthermore, the network layer tries to reduce the network's data traffic and overheads using optimization techniques. The application layer is the top layer that controls the services provided to the applications. This layer offers 
an interface to the user to control and manage the IoT devices. Furthermore, it provides services to the application depending on the nature of the application.

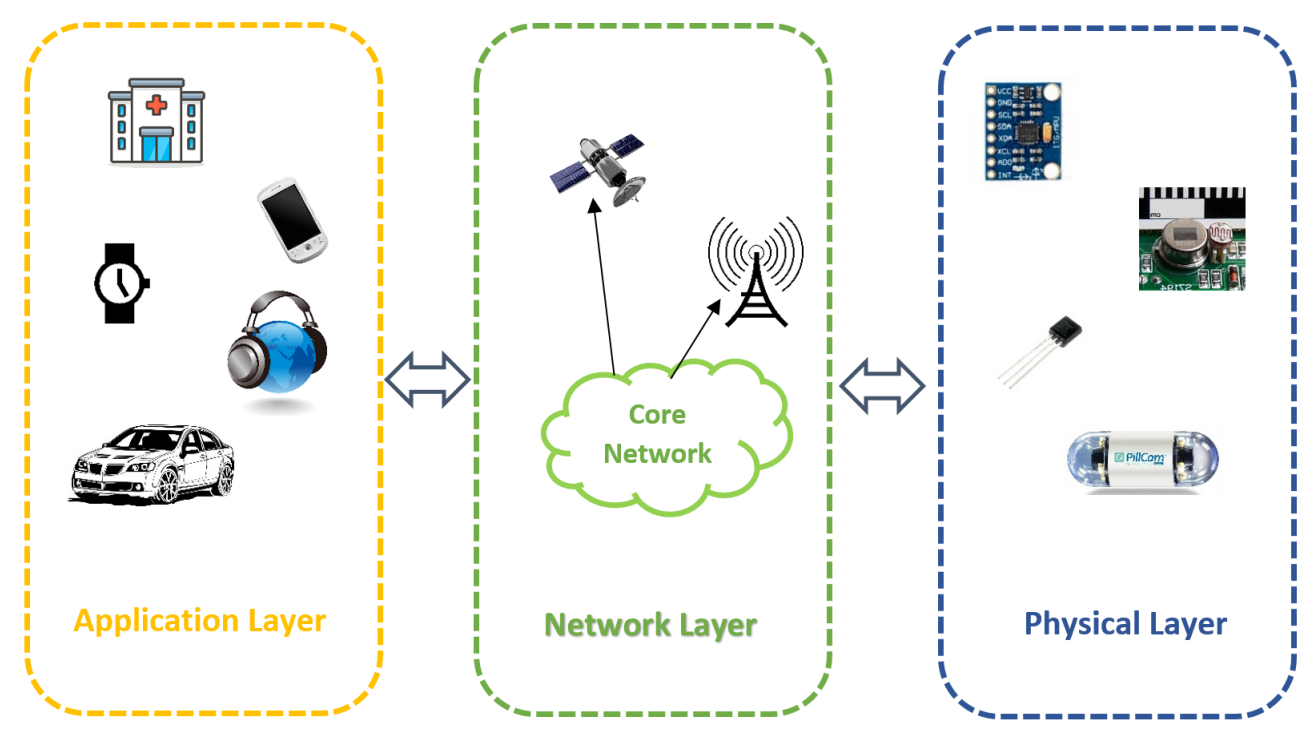

Figure 1. IoT architecture.

IoT is an extension of the Internet that envisions connecting all daily devices to the Internet for communications through interactions or sensing devices. These sensing devices are connected to form a network, termed a wireless sensor network (WSN) [9]. The IoT consisting of WSNs is essential for transforming the world into a smart world [10]. According to the Cisco Annual Internet Report [11], the number of IoT devices will rise from 6.1 billion to 14.7 billion by the end of 2023. Among those 14.7 billion devices, more than $48 \%$ of them will assist users in performing daily tasks, such as home automation, security, and tracking applications. Therefore, they act as building blocks in smart cities, smart homes, smart transportation, smart healthcare, smart grids, and smart industry [12-16].

However, there exist numerous challenges in the development of such IoT applications. For example, health-related applications require rapid and reliable data transmission. Monitoring applications in smart environments require energy-efficient and robust protocols [17]. Similarly, there are challenges associated with cost, connectivity, and hardware limitations [18]. However, localization is one key challenges that needs to be addressed in the majority of smart applications. It is the process of acquiring an object or user's location through intelligent devices (sensors) in an indoor or outdoor environment. It is a critical requirement in most smart applications $[19,20]$. An exponential increase in smartphones, wristwatches, and other intelligent wireless IoT devices is motivating researchers to develop efficient localization schemes. As a result, we are witnessing a significant rise in localization schemes intended to operate in healthcare, agriculture, environmental work, and habitat monitoring [21-23]. Therefore, this paper investigates the IoT-based localization and the proposed schemes $[24,25]$. The overall contributions of the paper are listed as follows.

1. It provides an overview of localization and its key performance indicators (KPIs).

2. It provides a comprehensive and thorough survey of the recent indoor and outdoor localization schemes. In addition, it highlights the aim and purpose of each localization scheme.

3. It evaluates each scheme with different KPIs such as localization accuracy, energy efficiency, target prediction, target recovery, and security. This evaluation is beneficial for readers aiming to develop a specific localization application.

4. The analysis presents a discussion on IoT localization and highlights the challenges faced by IoT-based localization.

5. It provides open research issues for the researchers working in the localization domain. 
A list of acronyms is given in Table 1 . The rest of the paper is organized as follows. Section 2 discusses localization and its KPIs. The overall methodology for paper selection is explained in Section 3. Section 4 provides a detailed review of select papers. The discussion is in Section 5. Finally, Sections 6 and 7 present the future directions and the conclusion, respectively.

Table 1. List of important acronyms.

\begin{tabular}{|c|c|c|c|}
\hline Acronym & Extended Meaning & Acronym & Extended Meaning \\
\hline WSN & Wireless Sensor Network & $\mathrm{SN}$ & Sensor Node \\
\hline $\mathrm{AN}$ & Anchor Nodes & IoT & Internet of Things \\
\hline $\mathrm{NH}$ & Neighbourhood Heuristics & ETX-NH & Expected Transmissions with Neighbourhood Heuristics \\
\hline PF-DLSTA & $\begin{array}{l}\text { Particle Filtering based Dynamic Lookahead Tree Based } \\
\text { Tracking Algorithm }\end{array}$ & WSHAN & Wireless Sensor Hole Aware Network \\
\hline BCTT & Boundary Static Clustering Target Tracking & $\mathrm{SCDCH}$ & Static Cluster and Dynamic Cluster Head \\
\hline KPI & Key Performance Indicator & ML & Machine Learning \\
\hline $\begin{array}{l}\text { PPHD- } \\
\text { MMA }\end{array}$ & $\begin{array}{l}\text { Particle filter based Probability Hypothesis Density } \\
\text { incorporated with Multipath-to-Measurement Association }\end{array}$ & VGTR & Virtual Grid-based Target Recovery \\
\hline FSM & Fuzzy Sensing Model & DCTC & Dynamic Convoy Tree-based Collaboration \\
\hline RFID & Radio frequency identification & GPS & Global Positioning System \\
\hline JPDA & Joint Probablistic Data Association & PUESRF & Particle wise Update version of Ensemble Square Root Filter \\
\hline IPAH & Improved Prediction based Adaptive-Head & DCTT & Distributed Cluster-based algorithm for Target Tracking \\
\hline PCTT & Prediction-based Clustering algorithm for Target Tracking & PSO & Particle Swarm Optimization \\
\hline ASMT & Augmented State-based Multi-target Tracking & GTPM & Game Theory Payoff Matrix \\
\hline SRPTT & Secure and Reliable Prediction-based Target Tracking Protocol & AEKF & Adaptive Extended Kalman Filter \\
\hline DHSCA & Dual Head Static Clustering Algorithm & $\mathrm{ACDF}$ & Adaptive Consensus based with the Distributed estimator Filter \\
\hline UKF & Unscented Kalman Filter & 4WS4WD & Four-wheel-steering and four-wheel-driving \\
\hline HNTA & Hybrid Network Tracking Algorithm & POMDP & Partially Observed Markov Decision Process \\
\hline EEA-IAH & Energy-Aware Improved Adaptive-Head & LPPT & Low Prediction Precision requirement Target tracking \\
\hline CLTA & Cooperative Localization and Tracking Algorithm & FTS & Fuzzy Tree System \\
\hline DMGIF & Distributed Multiple Gaussian Information Filter & ARIMA & Auto Regressive with Moving Average \\
\hline ELM & Extreme Learning Machine & LEMon & Localization Employing a location-unaware MObile unit \\
\hline RSSI & Received Signal Strength Indication & LEMon-M & LEMOn for localization Matching \\
\hline TS PM-PHD & Two Steps Product Multi-sensor Probability Hypothesis Density & MC-SSN & Mission Critical Sensors and Sensor Networks \\
\hline $\begin{array}{c}\text { AIE- } \\
\text { MCMCDA }\end{array}$ & $\begin{array}{l}\text { Augmented Input Estimation Markov Chain Monte-Carlo } \\
\text { Data Association }\end{array}$ & CS-BnB & Convex hull Sampling based Branch and Boun \\
\hline AMCL & Adaptive Monte Carlo Localization & IMM & Interactive Multi Model \\
\hline
\end{tabular}

\section{Key Performance Indicators in Localization}

Localization is one of the essential applications of smart IoT. Sensor nodes (SNs) keep track of the target and report the location to the user's application in localization. It requires a single $\mathrm{SN}$ or the collaboration of multiple SNs for the tracking of an object. In general, localization is divided into two types: range-based and range-free localization. Range-based localization schemes calculate the distance using multiple geometric and cross grid overlapping techniques such as received signal strength indicator (RSSI), angle of arrival (AoA), and time of arrival (ToA). The anchor nodes' (AN) positions are known in this technique, and SN determines their locations with reference to AN [26]. In contrast, range-free schemes use hop-count and distance between AN to SN. Furthermore, it uses geometric methods and estimation for localization [27].

A single $\mathrm{SN}$ often causes rapid energy depletion, high computation, and low accuracy, which can be resolved by employing multiple SNs [28]. The IoT unifies multiple transmission techniques, such as Zigbee, Bluetooth, Infrared, $\mathrm{WiFi}$, and the Internet, for efficient target localization and tracking. The choice of SNs may vary concerning applications such as radio frequency identification (RFID) in indoor tracking and acoustic sensors array for underwater localization. This inter-linkage and smooth cooperation among IoT devices is the cornerstone for its efficient and reliable working. Hence, the IoT-based network is very heterogeneous in SNs and communication protocols [29,30]. It gives rise to several chal- 
lenges due to non-standardization or unifying of different protocols. However, depending on the target application, these SNs have several KPIs: energy consumption, rapid power depletion, computation, and security. The scope of this review is to highlight and evaluate only the localization KPIs in IoT devices. Therefore, we have not considered KPIs related to other IoT applications. The critical KPIs of target localization are illustrated in Figure 2.

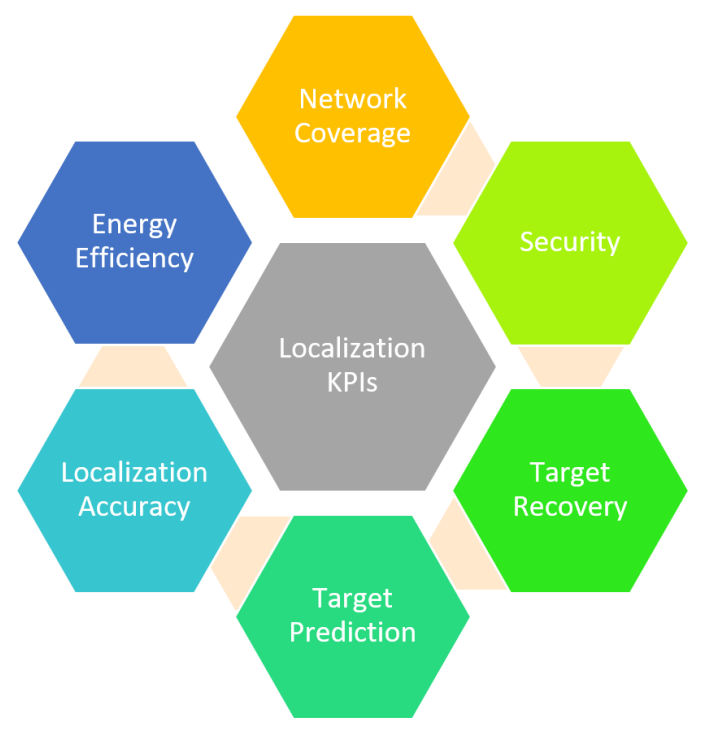

Figure 2. Parameter affecting the performance of localization.

\subsection{Network Coverage}

Coverage is associated with the sensing range of the WSN. In localization, it is the extent of a target in a network under the surveillance of a SN. Therefore, it ensures the uniformity of the SNs and avoids black holes in the overall network.

\subsection{Security}

Generally, the WSN is deployed in an external environment such as a forest, a building, or a battlefield. It transmits information over the wireless channel vulnerable to various security attacks such as jamming, spoofing, misdirection, and Sybil attacks. Therefore, security ensures information reliability, authentication, and integrity, and avoids security attacks such as flooding and spoofing.

\subsection{Target Recovery}

Sometimes, a target gets lost due to a prediction error, a communication error, a black hole, or an SN failure. It leads a target to pass through a specific region undetected and compromise overall tracking accuracy. Target recovery tries to retrieve such target(s) in minimum time using an optimal number of sensors to ensure overall energy efficiency.

\subsection{Target Prediction}

The prediction in localization aims to predict the location of the mobile target. It results in improving the efficiency of target localization. In general, this parameter measures the overall probabilities of true-positive and false-negative results.

\subsection{Localization Accuracy}

Localization accuracy specifies the accuracy by which the position of the target is determined. In localization, the location of the $\mathrm{SN}$ is critical, as a minor location error generates worthless data. 


\subsection{Energy Efficiency}

SNs run on batteries that are non-rechargeable and sometimes located in a nonchangeable environment [31]. Some SNs consume more energy during the localization due to idle listening, overhearing, and packet collision. The energy efficiency and lifetime of SNs pose severe issues in WSN, specifically in sensitive target tracking applications.

This paper aims to analyze the recent localization schemes based on the KPIs mentioned above. A detailed methodology of paper selection is presented in the next section.

\section{Methodology}

The Preferred Reporting Items for Systematic Reviews and Meta-Analyses (PRISMA) approach is used in this review [32]. An overview of the proposed methodology is presented in Figure 3. The overall methodology consists of four phases:

- Identification.

- Screening.

- Eligibility.

- Selection.
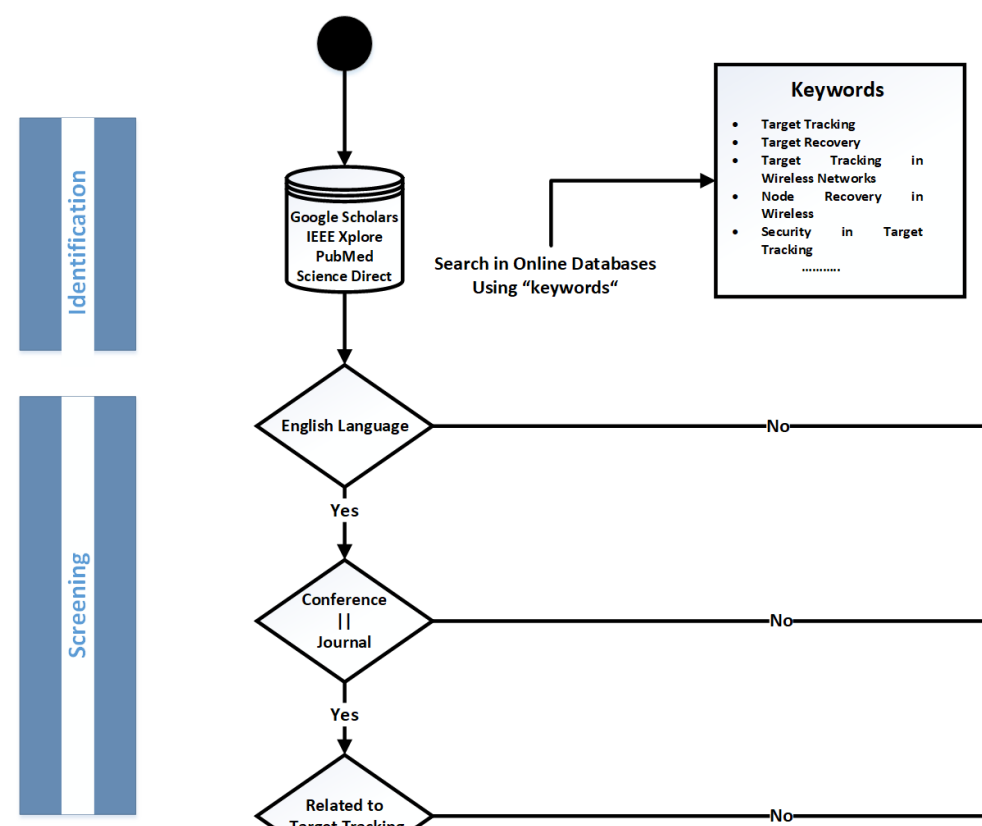<smiles>CCCC</smiles>
Using "keywords"
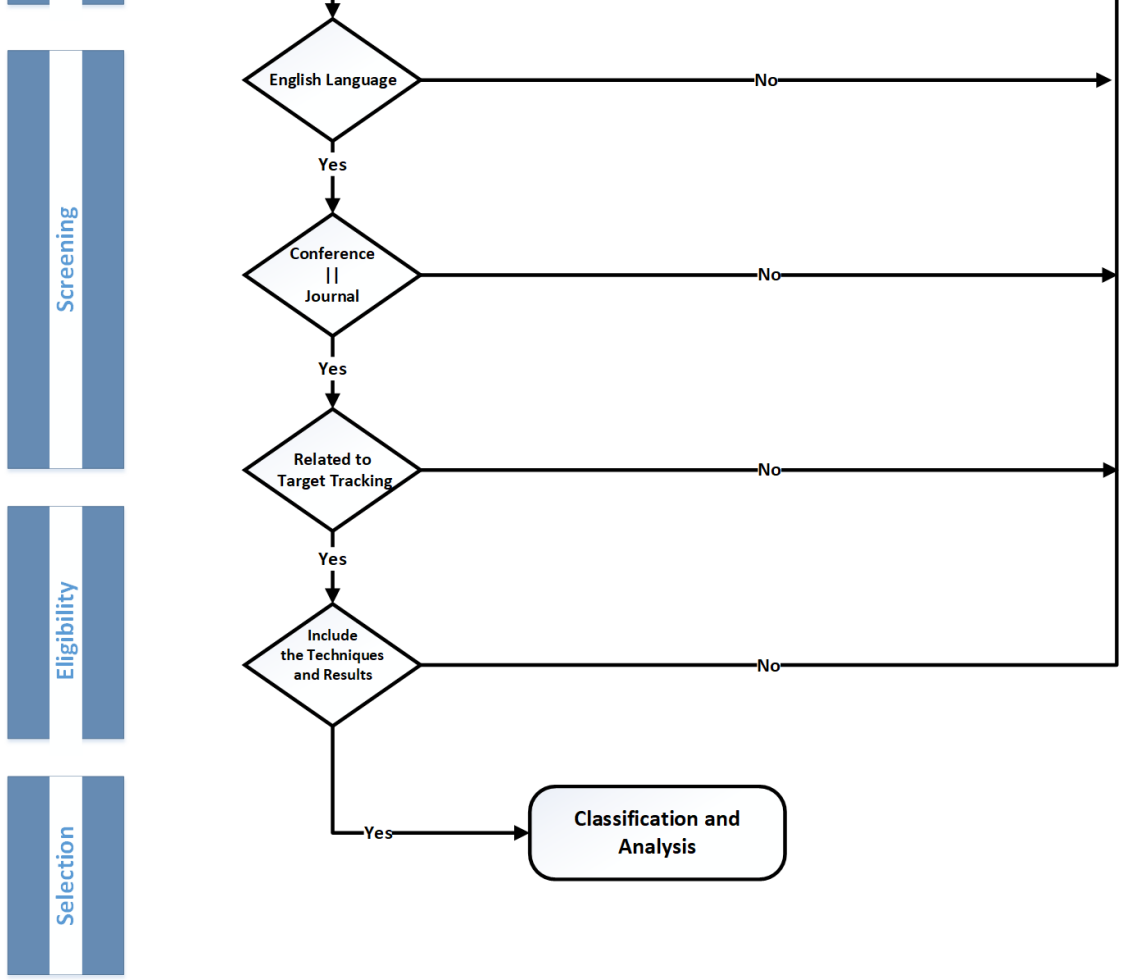

Figure 3. Proposed methodology.

The identification process consists of the initial selection of the papers based on the abstracts. Different science libraries, such as Google Scholar, IEEE Xplore, Pub Med, and Science Direct, were used using various strings. Table 2 provides the list of strings for searching desired papers. Based on the above-mentioned strings, more than 1000 
documents were obtained from multiple academic databases. Only documents newer than 2010 were considered in the identification process. The obtained documents were filtered out based on the title in the initial screening process. A total of 249 papers were selected after applying various screening filters, such as the English language. The 21 duplicates were also removed, which resulted in 228 documents for the eligibility check criteria. All the papers in this phase were read carefully, and the following parameters made them eligible for the selection process:

- The paper should be published in journal or conference.

- The papers should consider the target tracking.

- The papers should present a concrete methodology and results.

Table 2. Strings used in search engines.

\begin{tabular}{|c|c|}
\hline $\begin{array}{l}\text { Academic } \\
\text { Library }\end{array}$ & Search String \\
\hline $\begin{array}{l}\text { Google } \\
\text { Scholar }\end{array}$ & $\begin{array}{l}\Rightarrow \text { Target Tracking } \\
\Rightarrow \text { Target Localization in wireless Network } \\
\Rightarrow \text { Routing protocol for target tracking } \\
\Rightarrow \text { Target recovery in wireless networks } \\
\Rightarrow \text { Target Localization and artificial intelligence } \\
\Rightarrow \text { Single Target Tracking } \\
\Rightarrow \text { Multi Target Tracking } \\
\Rightarrow \text { Target Tracking Using Machine Learning } \\
\Rightarrow \text { Prediction Based target Tracking } \\
\Rightarrow \text { Energy Efficient Tracking } \\
\Rightarrow \text { Target Tracking Applications }\end{array}$ \\
\hline IEEE Xplore & 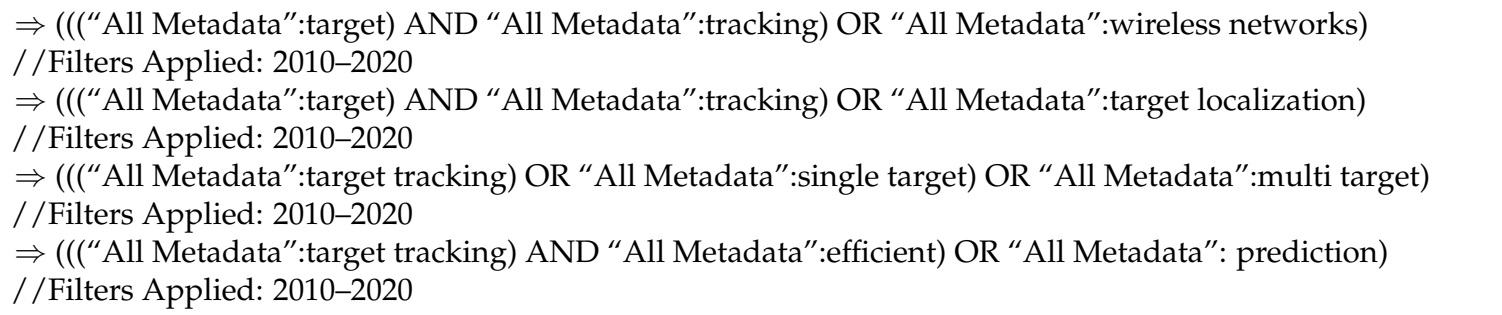 \\
\hline PubMed & $\begin{array}{l}\Rightarrow \text { target[Title/Abstract] AND tracking[Title/Abstract] OR wireless networks[Title/Abstract] AND } \\
\text { ("2010/01/01"[PDAT]: “2020/01/01”[PDAT]) } \\
\Rightarrow \text { target[Title/Abstract] AND tracking[Title/Abstract] OR efficient [Title/Abstract] AND } \\
\text { (“2010/01/01”[PDAT]: “2020/01/01"[PDAT]) } \\
\Rightarrow \text { target[Title/Abstract] AND tracking[Title/Abstract] OR machine learning [Title/Abstract] AND } \\
\text { (“2010/01/01"[PDAT]: “2020/01/01”[PDAT]) }\end{array}$ \\
\hline Science Direct & $\begin{array}{l}\Rightarrow \text { Target Tracking' and Wireless Networks or prediction. Limited to research articles, conference abstracts. } \\
\Rightarrow \text { Target Tracking' and Wireless Networks or efficient. Limited to research articles, conference abstracts. } \\
\Rightarrow \text { Target Tracking' and Wireless Networks or applications. Limited to research articles, conference abstracts. }\end{array}$ \\
\hline
\end{tabular}

Finally, a total of 40 papers were selected after eliminating the papers based on the above-mentioned criteria. These papers were used as a part of the review and further analyzed. The following section provides a complete analysis of selected publications using this methodology.

\section{Review of Location Aware Schemes in IoT}

A comparison of the state-of-the-art studies is given in Table 3. From the table, it is clear that most of the existing surveys target a particular localization domain, i.e., outdoor or indoor.

The main focuses of already published surveys were accuracy, energy efficiency, target prediction, and security. They lack some critical KPIs, such as recovery, prediction, security, and localization with smart gadgets, i.e., smartphones. Only $28 \%$ of publications covered prediction; $35 \%$ covered security and localization with smartphones. Simultaneously, no 
published survey covered the target recovery KPI, which is an important indicator that affects the overall performance of the IoT-based localization scheme.

Furthermore, these surveys lacked detail and generic discussion in terms of protocols and techniques for localization schemes. Therefore, there is a need for a cross-domain survey that puts forward an in-depth discussion on the IoT-based localization scheme. As mentioned in Section 3, a total of 40 papers were selected using the PRISMA approach. The key ideas of the selected papers are presented in Table 4. In addition to that, the selected studies were analyzed based on publishing details and target applications.

Table 3. Comparison between this paper and published surveys.

\begin{tabular}{|c|c|c|c|c|c|c|c|c|}
\hline References & $\begin{array}{c}\text { Indoor } \\
\text { Localization }\end{array}$ & $\begin{array}{c}\text { Outdoor } \\
\text { Localization }\end{array}$ & $\begin{array}{l}\text { Smartphone } \\
\text { Localization }\end{array}$ & Security & $\begin{array}{c}\text { Energy } \\
\text { Efficiency }\end{array}$ & Accuracy & $\begin{array}{c}\text { Target } \\
\text { Recovery }\end{array}$ & $\begin{array}{c}\text { Target } \\
\text { Prediction }\end{array}$ \\
\hline [33] & $\checkmark$ & & & & $\checkmark$ & $\checkmark$ & & \\
\hline [34] & $\checkmark$ & & & & $\checkmark$ & $\checkmark$ & & \\
\hline [35] & $\checkmark$ & & & & $\checkmark$ & $\checkmark$ & & \\
\hline [36] & $\checkmark$ & & & $\checkmark$ & $\checkmark$ & $\checkmark$ & & $\checkmark$ \\
\hline [37] & $\checkmark$ & & & & & $\checkmark$ & & \\
\hline [38] & $\checkmark$ & & & $\checkmark$ & & $\checkmark$ & & \\
\hline [39] & & $\checkmark$ & & & $\checkmark$ & $\checkmark$ & & \\
\hline [40] & $\checkmark$ & $\checkmark$ & & & $\checkmark$ & $\checkmark$ & & $\checkmark$ \\
\hline [41] & $\checkmark$ & $\checkmark$ & & & & $\checkmark$ & & \\
\hline [42] & $\checkmark$ & $\checkmark$ & & $\checkmark$ & & $\checkmark$ & & \\
\hline [43] & $\checkmark$ & & $\checkmark$ & $\checkmark$ & $\checkmark$ & $\checkmark$ & & \\
\hline [44] & $\checkmark$ & & $\checkmark$ & & & $\checkmark$ & & \\
\hline [45] & $\checkmark$ & $\checkmark$ & $\checkmark$ & & & $\checkmark$ & & $\checkmark$ \\
\hline [46] & $\checkmark$ & & $\checkmark$ & & $\checkmark$ & $\checkmark$ & & $\checkmark$ \\
\hline Ours & $\checkmark$ & $\checkmark$ & $\checkmark$ & $\checkmark$ & $\checkmark$ & $\checkmark$ & $\checkmark$ & $\checkmark$ \\
\hline
\end{tabular}

Table 4. Key ideas of selected papers.

\section{Name}

Delaney et al. [47]

Alaybeyoglu et al. [48]

Mirsadeghi et al. [49]

Patil et al. [50]

Rouhani et al. [51]

Wahdan et al. [52]

Zhou et al. [53]

\section{Overview}

This paper presents an energy efficient routing protocol using NHs model for tree structured WSN. Apart from energy efficiency, the proposed solution has the ability to present good results in a lossy network environment.

This paper presents an efficient tracking scheme for high speed targets. Additionally, the proposed scheme helps in reducing the target miss ratio during the whole tracking lifecycle.

This paper presents an energy efficient prediction based target tracking scheme for WSN. The node closest to the object or with the highest energy is selected as a $\mathrm{CH}$ to prolong the network lifetime.

This paper presents an energy efficient WSHAN to improve the efficiency of target tracking target recovery.

This paper presents a solution to resolve the boundary target tracking issues using static clustering. The proposed solution is energy efficient, reasonably accurate and reliable in terms of target tracking.

This paper presents a hybrid solution of static networking clustering and dynamic $\mathrm{CH}$. The dynamic $\mathrm{CH}$ uniformly utilize the energy of member SNs to prolong the network lifetime and prediction.

This paper presents a fusion of MMA and PPHD for multi-target tracking in an urban area. Additionally, K-mean clustering is used to calculate the number of targets at any given time. The proposed scheme results in the tracking of dynamically changing unknown numbers of targets in urban areas. 
Table 4. Cont.

\begin{tabular}{|c|c|}
\hline Name & Overview \\
\hline Amudha et al. [54] & $\begin{array}{l}\text { This paper presents a multi camera based scheme for target tracking. In this scheme, the camera near } \\
\text { the mobile target is activated while all other cameras remain in a sleep state to conserve energy. } \\
\text { In contrast, all the cameras are activated when a target is lost to improve the tracking. }\end{array}$ \\
\hline Bhowmik et al. [55] & $\begin{array}{l}\text { This paper presents an algorithm is to improve the overall coverage and target tracking. In addition } \\
\text { to that, the proposed algorithm uses the FSM based RSSI tracking algorithm to make it more } \\
\text { energy efficient. }\end{array}$ \\
\hline Jinan et al. [56] & $\begin{array}{l}\text { This paper presents a multi-model framework based on the PUESRF and JPDA. It results in } \\
\text { improving the accuracy and precision of data that makes target tracking consistent. }\end{array}$ \\
\hline Darabkh et al. [57] & $\begin{array}{l}\text { This paper presents an adaptive } \mathrm{CH} \text { algorithm with an aim to achieve a better target tracking by } \\
\text { efficiently electing } \mathrm{CH} \text { and cluster members. The proposed algorithm is energy efficiency and } \\
\text { improves the network scalability. }\end{array}$ \\
\hline Khakpour et al. [58] & $\begin{array}{l}\text { This paper presents a fusion of DCTT and PCTT against vehicular tracking in a Vehicular Ad-hoc } \\
\text { Network. To improve the target prediction, The DCTT performs in a distributed manner while PCTT } \\
\text { is used for a centralized prediction algorithm. }\end{array}$ \\
\hline Joshi et al. [59] & $\begin{array}{l}\text { This paper presents a static cluster based target tracking for the prediction that is independent of } \\
\text { wireless network architecture (homogenous or heterogeneous). The proposed scheme uses a linear } \\
\text { prediction technique to calculate direction and speed to improve the target prediction. }\end{array}$ \\
\hline Xiao et al. [60] & $\begin{array}{l}\text { This paper presents a novel ASMT using Bayesian estimation to efficiently track multiple targets. } \\
\text { The ASMT provides high accuracy, precision based multi-target tracking, less computation and } \\
\text { solves the data association problem in WSN very efficiently by using location state and velocity state. }\end{array}$ \\
\hline Silva et al. [61] & $\begin{array}{l}\text { This paper presents an energy efficient scheme with the ability to detect and highlight the fake node } \\
\text { positioning and bogus data flooding. }\end{array}$ \\
\hline Oracevic et al. [62] & $\begin{array}{l}\text { This paper presents a SRPTT algorithm to prevent the rouge SN from faking its location or flooding } \\
\text { the bogus packets in a WSN. The SRPTT maintains a balance between security and mobile target } \\
\text { tracking by employing a reputation concept. }\end{array}$ \\
\hline Alshamaa et al. [63] & $\begin{array}{l}\text { This paper presents a novel zoning based localization technique for indoor target tracking. } \\
\text { The proposed technique develops a belief function by combining fingerprint based target observation } \\
\text { and evidence associated with sensor mobility to improve the accuracy of target tracking. }\end{array}$ \\
\hline Chen et al. [64] & $\begin{array}{l}\text { This paper presents an adaptive extended kalman filter to remove and update the noise covariance. } \\
\text { The proposed solution results in improving the accuracy and reliability of target tracking. }\end{array}$ \\
\hline Panag et al. [65] & $\begin{array}{l}\text { This paper presents a DHSCA to uniformly utilize sensors during the tracking. The proposed } \\
\text { algorithm simplifies the set-up phase time of the network resulting in reducing the overhead of } \\
\text { the network. }\end{array}$ \\
\hline Zhang et al. [66] & $\begin{array}{l}\text { This paper presents a dynamic clustering-based adaptive filtering scheme for target tracking in a } \\
\text { WSN. The proposed scheme consists of two stages hierarchal data aggregation technique, which } \\
\text { results in accurate and energy efficient target tracking. }\end{array}$ \\
\hline Qian et al. [67] & $\begin{array}{l}\text { This paper presents an AUKF algorithm to enhance the robustness and accuracy of the recovery } \\
\text { mechanism. The AUKF fine-tunes the noise covariance matrix to increase the accuracy and } \\
\text { robustness of the recovery mechanism. The vigorous scheduling of static and mobile SNs improves } \\
\text { the tracking probability with less energy consumption. }\end{array}$ \\
\hline Zhang et al. [68] & $\begin{array}{l}\text { This paper presents an algorithm based on a hybrid sensor network to estimate the target region via } \\
\text { static sensors. Additionally, a movement algorithm is presented for nodes to select the location. } \\
\text { The proposed solution results in conserving the energy by reducing the target tracking sensors. }\end{array}$ \\
\hline Li et al. [69] & $\begin{array}{l}\text { This paper presents a sensor selection technique based on POMDP to reduce the sensor selection } \\
\text { lagging. It results in improving the target tracking accuracy and reliability. }\end{array}$ \\
\hline Darabkh et al. [70] & $\begin{array}{l}\text { This paper presents an error and Energy-aware cluster head selection algorithm to improve the target } \\
\text { localization. The proposed algorithm improves energy consumption and simplifies the selection of } \\
\text { cluster members. Additionally, it reduces the packets overhead by minimizing the transmission of } \\
\text { control messages. }\end{array}$ \\
\hline Liu et al. [71] & $\begin{array}{l}\text { This paper presents energy efficient scheme with low prediction accuracy. Apart from energy } \\
\text { efficiency, it reduces the target miss rate probability. }\end{array}$ \\
\hline
\end{tabular}


Table 4. Cont.

\begin{tabular}{|c|c|}
\hline Name & Overview \\
\hline Luo et al. [72] & This paper presents a scheme to improve the target tracking for an indoor environment using a CLTA. \\
\hline Yu et al. [73] & $\begin{array}{l}\text { This paper presents a mobile node-based target tracking scheme to enhance the target tracking } \\
\text { accuracy and transmission reliability. }\end{array}$ \\
\hline Vallas et al. [74] & $\begin{array}{l}\text { This paper presents a Gaussian filter-based multi-sigma point filter to reduce the curse of } \\
\text { dimensionality in high dimension systems. Furthermore, it improves the efficiency of tracking the } \\
\text { multiple targets in a WSN. }\end{array}$ \\
\hline Ghodousi et al. [75] & $\begin{array}{l}\text { This paper presents an energy efficient tracking scheme using ARIMA and UKF. The ARIMA, after } \\
\text { observing target in equal interval, predicts its future location while UKF estimates the target location. } \\
\text { The proposed scheme preserves the energy of SNs and improves the network lifetime. }\end{array}$ \\
\hline Liang et al. [76] & This paper presents a trust-based distributed KF scheme for secure and reliable target tracking. \\
\hline Khan et al. [77] & $\begin{array}{l}\text { This paper presents a dynamic clustering-based verifiable multi iteration scheme to improve target } \\
\text { tracking. The proposed scheme improves the accuracy and reliability of tracking. }\end{array}$ \\
\hline Liu et al. [78] & $\begin{array}{l}\text { This paper presents an object localization scheme to provide better localization results on the } \\
\text { sequences undergoing shape deformation and illumination changes. }\end{array}$ \\
\hline Nguyen et al. [79] & $\begin{array}{l}\text { This paper presents a solution to improve the accuracy of target tracking in harsh radio } \\
\text { environments. The proposed scheme is efficient in both indoor and outdoor environments. }\end{array}$ \\
\hline Ullah et al. [80] & $\begin{array}{l}\text { This paper presents an underwater target tracking scheme intending to achieve energy efficiency and } \\
\text { tracking accuracy. }\end{array}$ \\
\hline Alberto et al. [81] & $\begin{array}{l}\text { This paper presents a multi-model tracking system by unifying fingerprint-based tracking with } \\
\text { neural networks. The proposed system also employs a Gaussian outliers filter with neural networks } \\
\text { to further improve the tracking accuracy. }\end{array}$ \\
\hline Liu et al. [82] & $\begin{array}{l}\text { This paper presents a scheme for tracking multiple targets in a harsh environment accurately } \\
\text { and precisely. }\end{array}$ \\
\hline Liu et al. [83] & $\begin{array}{l}\text { This paper presents an AFS for accurate and efficient target tracking. The proposed scheme is robust } \\
\text { and fault-tolerant with a low target loss rate. Moreover, PSO is used to fine-tune and improve the } \\
\text { overall tracking performance. }\end{array}$ \\
\hline Mahmoudreza et al. [84] & $\begin{array}{l}\text { This paper presents a solution to tackle the multiple target tracking problems with accurate data } \\
\text { association. It results in the prevention of false alarms. }\end{array}$ \\
\hline Li et al. [85] & $\begin{array}{l}\text { This paper presents a hybrid solution to provide accurate and reliable localization in harsh } \\
\text { manufacturing workshops. }\end{array}$ \\
\hline Reisinger et al. [86] & This paper presents an IMM tracking scheme unified with UKF to track the targets efficiently. \\
\hline
\end{tabular}

\subsection{Distribution Based on Publishing Year}

We aim to highlight the recent trends in target localization. Therefore, the papers from the last seven years (2014 and onward) are considered. The yearly distribution of the selected publications is presented in Figure 4. From the figure, it is depicted that there was less interest in localization in earlier years. However, it started rising from 2017. The last three years of research (2017-2019) comprised 67\% of publications selected for this review. 2020 was just beginning when the papers were shortlisted. However, based on the trend, more contributions are expected in this domain than in past years.

\subsection{Distribution Based on Publication Venue}

This section aims to highlight the publication venue distribution. Our study includes various publication venues, such as IEEE, Elsevier, MDPI, and SAGE. The distribution of publications concerning the venues is presented in Figure 5. It was found that IEEE and Elsevier support most publications in the domain of target tracking- $57.5 \%$ and $20 \%$, respectively. Therefore, these two venues are recommended for localization in IoT. 


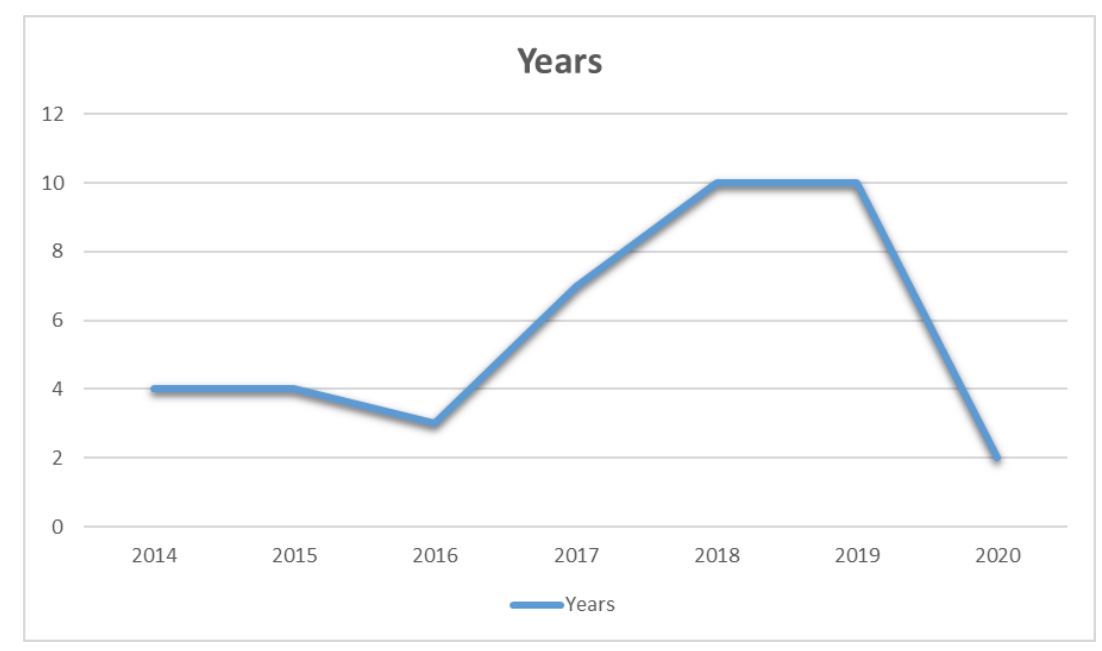

Figure 4. Distribution based on publication year.

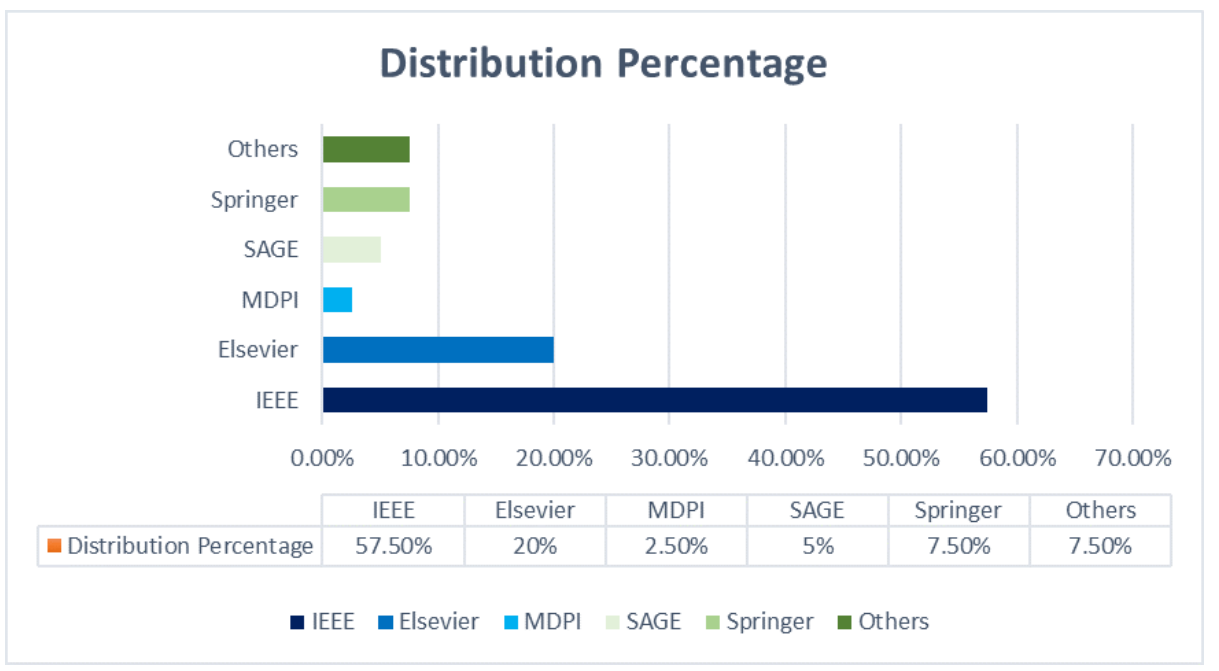

Figure 5. Distribution based on publishing venue.

\subsection{Distribution Based on Publication Type}

As mentioned in Section 3, we have only considered conference proceedings and journal articles for the review. The distribution percentages of those publication types are presented in the form of a pie chart. From the Figure 6, it is clear that our results are mainly backed up by journal articles $(67 \%)$.

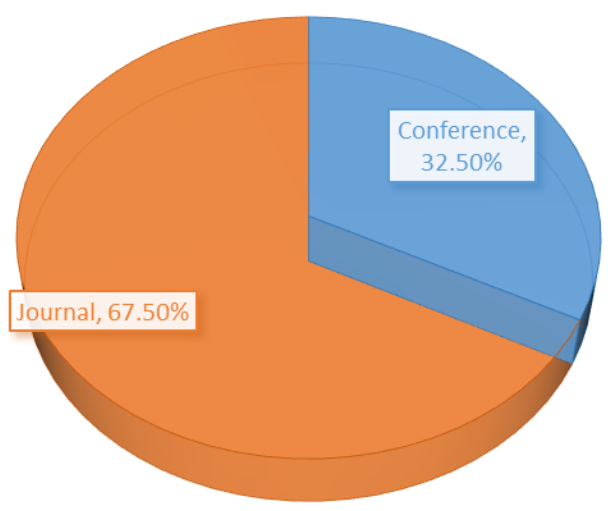

Figure 6. Distribution based on publication type. 


\subsection{Analysis Based on Localization KPIs}

There are various localization KPIs, such as energy efficiency, localization accuracy, target prediction, target recovery, and security. Every paper is trying to address single or multiple KPIs. Table 5 lists the selected publications along with the target KPIs. The lifetime of sensors is a major concern in WSNs, as battery replacement is a tiring and timeconsuming job. Additionally, low-battery or abandoned sensors can halt the performance of the overall network in emergencies.

Furthermore, accurate predictions and identification of targets are desired in locationaware schemes. Therefore, energy efficiency, tracking accuracy, and target prediction are the most researched KPIs in target tracking of WSNs, as shown in Figure 7. In contrast, target recovery and security were explored in only $11 \%$ of the papers selected. These are also important aspects of localization that need the researcher's attention in the future. In addition to the above analysis, a general overview of all the papers, including the proposed approach, network structure, number of targets, and performance parameters, is presented in Table 6.

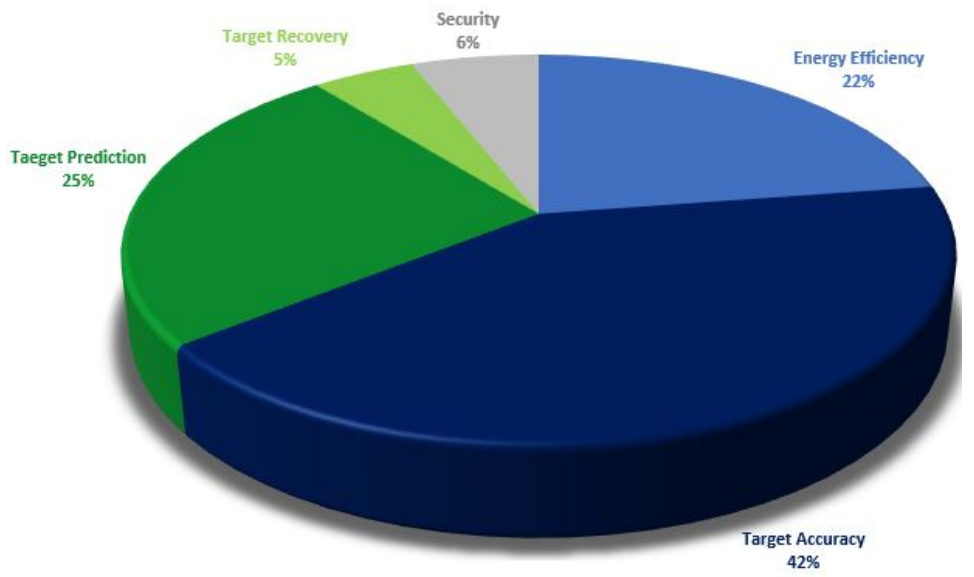

Figure 7. Distribution based on target challenges.

Table 5. KPIs Addressed in Selected Papers.

\begin{tabular}{|c|c|c|c|c|c|}
\hline Name & Energy Efficiency & Localization Accuracy & Target Predication & Target Recovery & Security \\
\hline Delaney et al. [47] & $\checkmark$ & & & & \\
\hline Alaybeyoglu et al. [48] & $\checkmark$ & $\checkmark$ & $\checkmark$ & & \\
\hline Mirsadeghi et al. [49] & $\checkmark$ & $\checkmark$ & $\checkmark$ & & \\
\hline Patil et al. [50] & $\checkmark$ & $\checkmark$ & $\checkmark$ & $\checkmark$ & \\
\hline Rouhani et al. [51] & $\checkmark$ & $\checkmark$ & & & \\
\hline Wahdan et al. [52] & $\checkmark$ & $\checkmark$ & $\checkmark$ & & \\
\hline Zhouet al. [53] & & $\checkmark$ & $\checkmark$ & & \\
\hline Amudha et al. [54] & $\checkmark$ & $\checkmark$ & & $\checkmark$ & \\
\hline Bhowmik et al. [55] & $\checkmark$ & $\checkmark$ & & & \\
\hline Jinan et al. [56] & & $\checkmark$ & $\checkmark$ & & \\
\hline Darabkh et al. [57] & $\checkmark$ & $\checkmark$ & $\checkmark$ & & \\
\hline Khakpour et al. [58] & & $\checkmark$ & $\checkmark$ & & \\
\hline Joshi et al. [59] & & $\checkmark$ & $\checkmark$ & & \\
\hline Xiao et al. [60] & & $\checkmark$ & & & \\
\hline Silva et al. [61] & $\checkmark$ & & $\checkmark$ & & $\checkmark$ \\
\hline
\end{tabular}


Table 5. Cont.

\begin{tabular}{|c|c|c|c|c|c|}
\hline Name & Energy Efficiency & Localization Accuracy & Target Predication & Target Recovery & Security \\
\hline Oracevic et al. [62] & & $\checkmark$ & $\checkmark$ & & $\checkmark$ \\
\hline Alshamaa et al. [63] & & $\checkmark$ & & & \\
\hline Chen et al. [64] & & $\checkmark$ & $\checkmark$ & & \\
\hline Panag et al. [65] & $\checkmark$ & & & & \\
\hline Zhang et al. [66] & $\checkmark$ & $\checkmark$ & & & \\
\hline Qian et al. [67] & & & $\checkmark$ & $\checkmark$ & \\
\hline Zhang et al. [68] & $\checkmark$ & $\checkmark$ & & & \\
\hline Li et al. [69] & $\checkmark$ & & $\checkmark$ & & \\
\hline Darabkh et al. [70] & $\checkmark$ & $\checkmark$ & & & \\
\hline Liu et al. [71] & $\checkmark$ & $\checkmark$ & $\checkmark$ & & \\
\hline Luo et al. [72] & & $\checkmark$ & & & \\
\hline Yu et al. [73] & & $\checkmark$ & $\checkmark$ & & \\
\hline Vallas et al. [74] & & $\checkmark$ & $\checkmark$ & & \\
\hline Ghodousi et al. [75] & $\checkmark$ & $\checkmark$ & $\checkmark$ & & \\
\hline Liang et al. [76] & & $\checkmark$ & & & $\checkmark$ \\
\hline Khan et al. [77] & $\checkmark$ & $\checkmark$ & & & $\checkmark$ \\
\hline Liu et al. [78] & & $\checkmark$ & $\checkmark$ & & \\
\hline Nguyen et al. [79] & & $\checkmark$ & & & \\
\hline Ullah et al. [80] & $\checkmark$ & $\checkmark$ & & & \\
\hline Alberto et al. [81] & & $\checkmark$ & & & \\
\hline Liu et al. [82] & & $\checkmark$ & & & \\
\hline Liu et al. [83] & & $\checkmark$ & $\checkmark$ & $\checkmark$ & \\
\hline Mahmoudreza et al. [84] & & $\checkmark$ & & & $\checkmark$ \\
\hline Li et al. [85] & & $\checkmark$ & & & \\
\hline Reisinger et al. [86] & & $\checkmark$ & $\checkmark$ & & \\
\hline
\end{tabular}

Table 6. Overview of selected papers.

\begin{tabular}{llllll}
\hline Ref. & Proposed Approach & Network Structure & $\begin{array}{l}\text { Number of } \\
\text { Targets }\end{array}$ & $\begin{array}{l}\text { Performance } \\
\text { Parameters }\end{array}$ & Tool \\
\hline$[47]$ & ETX-NH & Tree & Single & PDR: $96 \%$ & TOSSIM \\
\hline$[48]$ & PF-DLSTA & Tree & Single & N/A & NS2 \\
\hline$[49]$ & Low Power Target Prediction & Dynamic Cluster & Single & MR: $0.69 \%$ & N/A \\
\hline Mechanism & WSHAN & Dynamic Cluster & Single & EE: $37 \%$ & MATLAB \\
\hline$[51]$ & BCTT & Static Cluster & Single & EE: $48 \%$ & Omnet++ \\
\hline$[52]$ & SCDCH & Static Cluster & Single & N/A & MATLAB \\
\hline$[53]$ & PPHD-MMA & Dynamic Cluster & Multiple & N/A & N/A \\
\hline$[54]$ & VGTR & Dynamic Cluster & Single & TMR: $99 \%$ reduction & MATLAB \\
\hline$[55]$ & DCTC with Fuzzy Sensing & Tree & Single & N/A & TinyOS and nesC \\
\hline$[56]$ & JPDA, PUESRF & Dynamic Cluster & Multiple & N/A & N/A \\
\hline
\end{tabular}


Table 6. Cont.

\begin{tabular}{|c|c|c|c|c|c|}
\hline Ref. & Proposed Approach & Network Structure & $\begin{array}{l}\text { Number of } \\
\text { Targets }\end{array}$ & $\begin{array}{l}\text { Performance } \\
\text { Parameters }\end{array}$ & Tool \\
\hline [57] & IPAH & Dynamic Cluster & Single & $\begin{array}{l}\text { EE: } 40 \% \text { improved, } \\
\text { LE: } 52 \% \text { improved }\end{array}$ & MATLAB \\
\hline [58] & DCTT, PCTT & Static Cluster & Single & N/A & NS2 + TOSSIM \\
\hline [59] & $\begin{array}{l}\text { Prediction based object } \\
\text { tracking algorithm }\end{array}$ & Static Cluster & Single & PA: $99 \%$ & NS2 \\
\hline [60] & ASMT & Static Cluster & Multiple & FR: $>14 \%$ & $\mathrm{~N} / \mathrm{A}$ \\
\hline [61] & GTPM & Dynamic Cluster & Single & N/A & NS2 \\
\hline [62] & SRPTT & Static Cluster & Single & $\mathrm{N} / \mathrm{A}$ & Java Simulator \\
\hline [63] & $\begin{array}{l}\text { Extended observation model, } \\
2^{\text {nd }} \text { mobility model }\end{array}$ & Static Cluster & Single & $\mathrm{N} / \mathrm{A}$ & $\mathrm{N} / \mathrm{A}$ \\
\hline [64] & AEKF & Static Cluster & Single & RMSE: $32.53 \%$ & N/A \\
\hline [65] & DHSCA & Static Cluster & Single & $\mathrm{N} / \mathrm{A}$ & $\begin{array}{l}\text { Fortran } \\
\text { PowerStation } 4.0\end{array}$ \\
\hline [66] & $\mathrm{ACDF}$ & Dynamic Cluster & Single & $\mathrm{N} / \mathrm{A}$ & $\mathrm{N} / \mathrm{A}$ \\
\hline [67] & AUKF & Static Cluster & Single & $\mathrm{N} / \mathrm{A}$ & MATLAB \\
\hline [68] & HNTA & Hybrid Cluster & Multiple & $\mathrm{N} / \mathrm{A}$ & N/A \\
\hline [69] & $\begin{array}{l}\text { Adaptive sensor selection } \\
\text { algorithm with POMDP }\end{array}$ & Dynamic Cluster & Multiple & $\mathrm{N} / \mathrm{A}$ & $\mathrm{N} / \mathrm{A}$ \\
\hline [70] & EEA-IAH & Dynamic Cluster & Single & N/A & MATLAB \\
\hline [71] & LPPT & Static Cluster & Single & $\begin{array}{l}\text { Reduce MR: } 36.34 \% \text {, } \\
\text { EE: } 5.2 \text { times }\end{array}$ & Omnet++ \\
\hline [72] & CLTA & Dynamic Cluster & Single & LE: $0.65 \mathrm{~m}$ & MATLAB \\
\hline [73] & FTS & Tree & Single & LE: >50 improvement & MATLAB \\
\hline [74] & DMGIF & Dynamic Cluster & Multiple & $\mathrm{N} / \mathrm{A}$ & $\mathrm{N} / \mathrm{A}$ \\
\hline [75] & ARIMA, AUKF & Dynamic Cluster & Single & $\mathrm{N} / \mathrm{A}$ & $\begin{array}{l}\text { Opnet }+ \\
\text { MATLAB }\end{array}$ \\
\hline [76] & $\begin{array}{l}\text { Trust-based distributed } \\
\text { Kalman filtering. }\end{array}$ & Dynamic Cluster & Single & $\mathrm{N} / \mathrm{A}$ & $\mathrm{N} / \mathrm{A}$ \\
\hline [77] & $\begin{array}{l}\text { Dynamic cooperative } \\
\text { multilateral sensing }\end{array}$ & Dynamic Cluster & Single & LE: $19 \%$ improved & MATLAB \\
\hline [78] & ELM compressive sensing & Dynamic Cluster & Single & N/A & MATLAB \\
\hline [79] & LEMon, LEMon-M & Static Cluster & Single & $\begin{array}{l}\text { Outdoor and Indoor LE: } \\
10 \mathrm{~m} \text { and } 2 \mathrm{~m} \text { improved }\end{array}$ & $\mathrm{N} / \mathrm{A}$ \\
\hline [80] & $\begin{array}{l}\text { Distance and angle-based } \\
\text { localization }\end{array}$ & Dynamic Cluster & Single & $\begin{array}{l}\text { LE: } 90 \% \text { improved, } \\
\text { ABL: } 104.9 \mathrm{~m}\end{array}$ & $\mathrm{~N} / \mathrm{A}$ \\
\hline [81] & SWiBluX & Dynamic Cluster & Single & LE: $45 \%$ improved & $\mathrm{N} / \mathrm{A}$ \\
\hline [82] & TS PM-PHD & Dynamic Cluster & Multiple & $\mathrm{N} / \mathrm{A}$ & $\mathrm{N} / \mathrm{A}$ \\
\hline [83] & AFS for MC-SSN & Tree & Single & LE: $<0.2 \%$ & $\mathrm{~N} / \mathrm{A}$ \\
\hline [84] & AIE-MCMCDA & Dynamic Cluster & Multiple & LE: $0.39-4.12 \%$ & N/A \\
\hline [85] & CS-BnB, BnB-AMCL & Dynamic Cluster & Single & LE: $0.005 \mathrm{~m} / 0.111 \mathrm{deg}$ & 4WS4WDr \\
\hline [86] & IMM, UKF & Dynamic & Multiple & EE: 4 times & $\mathrm{N} / \mathrm{A}$ \\
\hline
\end{tabular}




\section{Discussion}

The above section provides a review of the papers selected. In addition to that, we have learned several lessons during our analysis. For example, a lot of research has been done in pursuance of making WSNs smart and energy-efficient. A system that consumes much energy is ill-suited for most applications. Therefore, energy consumption is one of the core issues in terms of smart environment and localization. It is directly linked to the latency and performance of localization. Some influential factors are the number of nodes and transmission range. The number of SNs involved in the target localization will significantly affect localization accuracy and energy consumption. The higher the involvement of SNs in localization, the higher the localization accuracy and energy consumption. Hence, we must maintain a balance between accuracy and overall energy consumption. The transmission range is also a leading cause of energy consumption. Higher signal power results in improving the signal range of the SN. However, it also results in quick energy depletion. Thus, a trade-off between signal range and energy consumption is required. Some other factors which affect energy consumption are interference and periodic beacon transmission.

Accuracy is fundamental in terms of localization and its applications. In recent years, extensive research has been done to improve localization accuracy. However, most of the schemes are environment-specific (indoor or outdoor), which affects their widespread applicability. In an indoor environment, the efficiency and accuracy of localization show notable reductions in the presence of objects, noise, and the multi-path effect. Hence, we need such schemes to limit the effect of surrounding objects and the multi-path impact on accuracy. Contrarily, the localization accuracy is primarily dependent on GPS and radio frequency (RF) in outdoor scenarios. However, high accuracy is not achievable, and batteries are depleted rapidly. The researchers are mainly focusing on indoor localization. Thus, outdoor localization is still an open issue. There is a need to design a scheme that is independent of the environment in terms of performance.

Different prediction algorithms are used to improve target localization. However, these power-hungry algorithms reduce the lifetimes of SNs. In recent years, researchers have presented different low duty cycle prediction schemes in which the SNs close to the target remain functional, while others are kept in a sleep state $[49,59]$. However, the prediction algorithm must be accurate because failure to accurately predict the next target location might drastically affect the localization accuracy and performance. The error in the prediction algorithm due to varying speed results in target loss. To recover the lost target, all SNs should go in an active state to track and recover the lost target. However, that results in the rapid depletion of energy. Hence, multiple energy-efficient recovery schemes have been presented using different filters such as KF, UKF [54,64]. Furthermore, some researchers have used the genetic algorithm for target tracking and recovery [83].

Security is also a major concern in mission-critical applications such as battlefield monitoring, soldier monitoring, and telehealth monitoring. Often these SNs are deployed in a hostile environment that can be easily corrupt and exploited by intruders. To overcome this, some authors proposed k-mean clustering for node authenticity in [76], in which only trustworthy SNs are used for localization and other operations. Some studies suggest the use of cryptography or digital signature base security [62,84]. Network coverage is one of the issues in WSN and directly related to localization performance. Non-uniform distribution of SNs would result in holes in the region of SN deployment. Multiple network techniques have been presented since. We differentiate network structures into two types: tree structure; cluster structure. In a tree-based network structure, deployed SNs form a logical tree architecture where data travel from leaf $\mathrm{SN}$ to root $\mathrm{SN}$. This process preserves energy by avoiding packet flooding and broadcasting.

However, all the nodes in a cluster structure are combined to form a cluster with one or multiple cluster heads (CHs). Cluster-based topology improves the scalability and bandwidth efficiency as compared to the other topologies. A $\mathrm{CH}$ reduces the packets transmitted to a base station, improving energy consumption, bandwidth usage, and security. Clustering can be either static or dynamic. Static clusters are created at network 
establishment time and remain fixed during the whole lifetime of the network. Apart from its simplicity, it has several drawbacks, such as the life of the whole cluster being dependent upon the $\mathrm{CH}$. Additionally, it eliminates the possibility of data sharing and collaboration among clusters. In contrast, dynamic clusters form on runtime as the target travels. They are more flexible and energy-efficient than static clustering, because the clusters are formed when the necessity arises. However, they face data redundancy and interference issues.

\section{Future Directions}

Localization and its applications have gained much attention from researchers in the past few years. Different schemes have been presented to improve energy efficiency, localization accuracy, target prediction, and security. Despite that, many aspects still need improvements to enhance the capabilities of these applications. In this section, we discuss the open research challenges and possible future directions in this domain.

- Environment Independence-The majority of studies focus on either indoor [87-90] or outdoor localization [91-93]. This environment-centric application's nature limits the applicability and widespread use in real-life scenarios. Hence, there is a dire need for environment-independent localization algorithms that are feasible for both indoor and outdoor applications [94-96]. This will eventually improve the adaptability of these applications in practical applications such as emergency evacuations, shipment/cargo tracking, and mission-critical applications.

- Security and Privacy-Security is one of the least explored challenges in IoT-based localization applications. However, this domain requires attention, as a user reveals far more personal information (via wireless channels) when using such applications. Compromising user location can be dangerous and life-threatening for some IoT services and applications, such as health, industry, and defense. For example, in an industrial environment, compromises in security and privacy might lead to a violation of confidential information related to the company's product. Henceforth, the rapid increase in cybersecurity challenges and lack of standardization for basic privacy mechanisms make it an open research problem [12,97]. Multiple authors have suggested the embedding of deep learning techniques to improve the security in IoT-based Localization applications [98-100]. Additionally, encryption algorithms and digital signatures using public and private keys can improve the resilience against external attacks $[101,102]$. However, the extent of security by using minimal resources is still a big challenge.

- Energy Efficiency - Generally, the SNs are tiny with irreplaceable batteries that make them resource-scarce. Due to this, energy consumption is one of the primary challenges in localization applications. For that, some energy-efficient techniques [103-105] have been presented. These SNs are mostly deployed in external environments, which motivates the use of energy harvesting [106,107]. In this context, a few energy harvesting techniques for the prolonging of network lifetime have been presented in the literature [108-111]. Furthermore, machine learning (ML) algorithms integrated with energy harvesting technology are also candidates for improving network lifetime and performance by predicting the amount of energy to be harvested from ambient in a specific duty cycle [112,113].

- Accuracy-Extensive research has been done on localization accuracy in IoT. However, most of the proposed schemes neglect the resource-scarce nature of the SN, thereby making them ineligible for real-life applications. For example, GPS and cellular data improve accuracy but deplete the battery rapidly. Additionally, the accuracy is compromised by the shadowing effect. Therefore, the implementations of error-resilient and vigorous mechanisms such as adaptive scheduling algorithms, prediction, and localization optimization schemes can be developed to improve localization accuracy [114]. Cloud computing with better prediction algorithms (process at cloud) can lead to accurate localization schemes while consuming minimal resources. In the literature, some authors proposed ML-based localization schemes [115-117] to improve 
the localization accuracy. By integrating ML with the localization, the progressive likelihood surpassed the posterior likelihood. In addition to that, this could also assist with predicting the target's next possible location. Henceforth, it will activate only those SNs which are closest to that prediction.

- Data Flow - The data flow varies from scenario to scenario, i.e., normal or emergency. During a natural catastrophe/disaster, communication is an essential part of an emergency evacuation. Likewise, IoT devices are commonly used in healthcare-e.g., remote monitoring $[118,119]$ and body posture monitoring $[120,121]$. The interruption in data flow can risk the patient's life, which makes IoT inadequate for the health sector. Therefore, such schemes need to be developed which can perform better in emergency scenarios [42,122]. Priority-driven approaches have the potential to overcome such problems. For example, the IEEE 802.15.6 WBAN standard and its compatible devices can resolve this problem. This standard consists of eight priority levels, and the data flow of emergency traffic is always prioritized [123].

- Data Association-Tracking multiple targets is a challenging task because of the differences in speed and direction of targets. Due to the presence of multiple targets, SNs receive multiple pieces of target information [124-126]. The main hurdle is to differentiate which information belongs to which target. This improbability in information results in the data association problem. Therefore, distinguishing the data of specific targets in the presence of multiple targets requires attention. Various classification algorithms, such as support vector machines (SVM), decision trees (DT), and neural networks offer the potential to resolve this problem.

\section{Conclusions}

IoT's intrinsic nature makes it deployable everywhere-e.g., roads, homes, forests, and even underwater. Localization is one of the widely used applications of IoT. It is used in diverse fields, such as healthcare, security surveillance, monitoring, and vehicle tracking. Localization offers application-oriented KPIs such as energy, target prediction, network coverage, and security. Therefore, numerous studies/schemes have been presented in the literature to address one or multiple KPIs. This paper presented a detailed review of the recently proposed localization schemes. The review examined different localization schemes, key ideas, propositions, network architectures, performance parameters, and target KPIs. It is noted that the dynamic $\mathrm{CH}$ selection improves the flexibility and energy efficiency of the scheme.

Furthermore, the paper showed that there is always a trade-off among various KPIs, i.e., target recovery and energy efficiency. The review also highlighted that most of the selected schemes improved the accuracy and energy efficiency of the localization applications. In contrast, security and target recovery were less explored. Lastly, we highlighted the open research challenges to improving the performance of localization in IoT.

Author Contributions: This study was conceptualized by M.A.K. and A.S. under the supervision of H.P. and H.-c.K.; M.A.K. and A.S. wrote all sections of the manuscript and H.P. and H.-c.K. conducted review and edited of it. All authors have read and agreed to the published version of the manuscript.

Funding: This work was supported by the National Research Foundation of Korea(NRF) grant funded by the Korea government(MSIT) (No. NRF-2019R1F1A1058951).

Institutional Review Board Statement: Not applicable.

Informed Consent Statement: Not applicable.

Data Availability Statement: Not applicable.

Conflicts of Interest: The authors declare no conflict of interest. 


\section{References}

1. Castellacci, F.; Viñas-Bardolet, C. Internet use and job satisfaction. Comput. Hum. Behav. 2019, 90, 141-152. [CrossRef]

2. Scheerder, A.J.; van Deursen, A.J.A.M.; van Dijk, J.A.G.M. Negative outcomes of Internet use: A qualitative analysis in the homes of families with different educational backgrounds. Inf. Soc. 2019, 35, 286-298. [CrossRef]

3. World Internet Users Statistics and 2020 World Population Stats. Internet World Stats. 2020. Available online: https://www. internetworldstats.com/stats.htm (accessed on 29 March 2021).

4. Yaqoob, I.; Hashem, I.A.T.; Ahmed, A.; Kazmi, S.A.; Hong, C.S. Internet of things forensics: Recent advances, taxonomy, requirements, and open challenges. Future Gener. Comput. Syst. 2019, 92, 265-275. [CrossRef]

5. Srinivasan, C.; Rajesh, B.; Saikalyan, P.; Premsagar, K.; Yadav, E. A review on the different types of internet of things (IoT). J. Adv. Res. Dyn. Control. Syst. 2019, 11, 154-158.

6. Jagannath, J.; Polosky, N.; Jagannath, A.; Restuccia, F.; Melodia, T. Machine learning for wireless communications in the Internet of Things: A comprehensive survey. Ad Hoc Netw. 2019, 93, 101913. [CrossRef]

7. Malik, H.; Alam, M.M.; Pervaiz, H.; Le Moullec, Y.; Al-Dulaimi, A.; Parand, S.; Reggiani, L. Radio resource management in NB-IoT systems: Empowered by interference prediction and flexible duplexing. IEEE Netw. 2019, 34, 144-151. [CrossRef]

8. Khan, S.Z.; Malik, H.; Sarmiento, J.L.R.; Alam, M.M.; Le Moullec, Y. Dorm: Narrowband iot development platform and indoor deployment coverage analysis. Procedia Comput. Sci. 2019, 151, 1084-1091. [CrossRef]

9. Kumar, D.P.; Amgoth, T.; Annavarapu, C.S.R. Machine learning algorithms for wireless sensor networks: A survey. Inf. Fusion 2019, 49, 1-25. [CrossRef]

10. Shafique, K.; Khawaja, B.A.; Sabir, F.; Qazi, S.; Mustaqim, M. Internet of Things (IoT) for Next-Generation Smart Systems: A Review of Current Challenges, Future Trends and Prospects for Emerging 5G-IoT Scenarios. IEEE Access 2020, 8, 23022-23040. [CrossRef]

11. Cisco Annual Internet Report-Cisco Annual Internet Report (2018-2023) White Paper. Available online: https://www. cisco.com/c/en/us/solutions/collateral/executive-perspectives/annual-internet-report/white-paper-c11-741490.html\#: \{\}: text=By\%202023\%2C\%20global\%20fixed\%20broadband,average\%20mobile\%20connection\%20by\%202023 (accessed on 29 March 2021).

12. Hassija, V.; Chamola, V.; Saxena, V.; Jain, D.; Goyal, P.; Sikdar, B. A Survey on IoT Security: Application Areas, Security Threats, and Solution Architectures. IEEE Access 2019, 7, 82721-82743. [CrossRef]

13. Hamdan, O.; Shanableh, H.; Zaki, I.; Al-Ali, A.R.; Shanableh, T. IoT-Based Interactive Dual Mode Smart Home Automation. In Proceedings of the 2019 IEEE International Conference on Consumer Electronics (ICCE), Las Vegas, NV, USA, 11-13 January 2019 ; pp. 1-2.

14. Trappey, A.J.C.; Trappey, C.V.; Govindarajan, U.H.; Sun, J.J.H. Patent Value Analysis Using Deep Learning Models-The Case of IoT Technology Mining for the Manufacturing Industry. IEEE Trans. Eng. Manag. 2019, 1-13. [CrossRef]

15. Saleem, Y.; Crespi, N.; Rehmani, M.H.; Copeland, R. Internet of Things-Aided Smart Grid: Technologies, Architectures, Applications, Prototypes, and Future Research Directions. IEEE Access 2019, 7, 62962-63003. [CrossRef]

16. Saboor, A.; Mustafa, A.; Ahmad, R.; Khan, M.A.; Haris, M.; Hameed, R. Evolution of Wireless Standards for Health Monitoring In Proceedings of the 2019 9th Annual Information Technology, Electromechanical Engineering and Microelectronics Conference (IEMECON), Jaipur, India, 13-15 March 2019; pp. 268-272.

17. Tang, J.; So, D.K.C.; Zhao, N.; Shojaeifard, A.; Wong, K. Energy Efficiency Optimization with SWIPT in MIMO Broadcast Channels for Internet of Things. IEEE Internet Things J. 2018, 5, 2605-2619. [CrossRef]

18. Zaidan, A.; Zaidan, B. A review on intelligent process for smart home applications based on IoT: Coherent taxonomy, motivation, open challenges, and recommendations. Artif. Intell. Rev. 2020, 53, 141-165. [CrossRef]

19. Rahman, A.; Li, T.; Wang, Y. Recent advances in indoor localization via visible lights: A survey. Sensors 2020, 20, 1382. [CrossRef]

20. Elbes, M.; Alrawashdeh, T.; Almaita, E.; AlZu'bi, S.; Jararweh, Y. A platform for power management based on indoor localization in smart buildings using long short-term neural networks. Trans. Emerg. Telecommun. Technol. 2020, e3867. [CrossRef]

21. Giri, A.; Dutta, S.; Neogy, S. Fuzzy logic-based range-free localization for wireless sensor networks in agriculture. In Advanced Computing and Systems for Security; Springer: Singapore, 2020; pp. 3-12.

22. McAllister, T.D.; El-Tawab, S.; Heydari, M.H. Localization of Health Center Assets Through an IoT Environment (LoCATE) In Proceedings of the 2017 Systems and Information Engineering Design Symposium (SIEDS), Charlottesville, VA, USA, 28 April 2017; pp. 132-137.

23. Song, Y.; Zhang, X.; Zhang, M.; Wang, J. Research of node localization algorithm based on wireless sensor networks in marine environment monitoring. J. Comput. Methods Sci. Eng. 2018, 18, 69-83. [CrossRef]

24. Ramnath, S.; Javali, A.; Narang, B.; Mishra, P.; Routray, S.K. IoT based localization and tracking. In Proceedings of the 2017 International Conference on IoT and Application (ICIOT), Nagapattinam, India, 19-20 May 2017; pp. 1-4.

25. Shit, R.C.; Sharma, S.; Puthal, D.; Zomaya, A.Y. Location of Things (LoT): A Review and Taxonomy of Sensors Localization in IoT Infrastructure. IEEE Commun. Surv. Tutor. 2018, 20, 2028-2061. [CrossRef]

26. Saeed, N.; Nam, H.; Al-Naffouri, T.Y.; Alouini, M.S. A state-of-the-art survey on multidimensional scaling-based localization techniques. IEEE Commun. Surv. Tutor. 2019, 21, 3565-3583. [CrossRef]

27. Shakshuki, E.; Elkhail, A.A.; Nemer, I.; Adam, M.; Sheltami, T. Comparative study on range free localization algorithms. Procedia Comput. Sci. 2019, 151, 501-510. [CrossRef] 
28. Lee, J.J.; Krishnamachari, B.; Kuo, C.C.J. Impact of energy depletion and reliability on wireless sensor network connectivity. Digit. Wirel. Commun. VI 2004, 5440, 169-180. [CrossRef]

29. Qiu, T.; Chen, N.; Li, K.; Atiquzzaman, M.; Zhao, W. How Can Heterogeneous Internet of Things Build Our Future: A Survey. IEEE Commun. Surv. Tutor. 2018, 20, 2011-2027. [CrossRef]

30. Farash, M.S.; Turkanović, M.; Kumari, S.; Hölbl, M. An efficient user authentication and key agreement scheme for heterogeneous wireless sensor network tailored for the Internet of Things environment. Ad Hoc Netw. 2016, 36, 152-176. [CrossRef]

31. Ramadan, K.F.; Dessouky, M.; Abd-Elnaby, M.; Abd El-Samie, F.E. Node-power-based MAC protocol with adaptive listening period for wireless sensor networks. AEU Int. J. Electron. Commun. 2018, 84, 46-56. [CrossRef]

32. Moher, D.; Liberati, A.; Tetzlaff, J.; Altman, D.G. Preferred reporting items for systematic reviews and meta-analyses: The PRISMA statement. Ann. Intern. Med. 2009, 151, 264-269. [CrossRef] [PubMed]

33. Zafari, F.; Gkelias, A.; Leung, K.K. A survey of indoor localization systems and technologies. IEEE Commun. Surv. Tutor. 2019, 21, 2568-2599. [CrossRef]

34. Yassin, A.; Nasser, Y.; Awad, M.; Al-Dubai, A.; Liu, R.; Yuen, C.; Raulefs, R.; Aboutanios, E. Recent advances in indoor localization: A survey on theoretical approaches and applications. IEEE Commun. Surv. Tutor. 2016, 19, 1327-1346. [CrossRef]

35. Oguntala, G.; Abd-Alhameed, R.; Jones, S.; Noras, J.; Patwary, M.; Rodriguez, J. Indoor location identification technologies for real-time IoT-based applications: An inclusive survey. Comput. Sci. Rev. 2018, 30, 55-79. [CrossRef]

36. Laoudias, C.; Moreira, A.; Kim, S.; Lee, S.; Wirola, L.; Fischione, C. A survey of enabling technologies for network localization, tracking, and navigation. IEEE Commun. Surv. Tutor. 2018, 20, 3607-3644. [CrossRef]

37. Liu, M.; Cheng, L.; Qian, K.; Wang, J.; Wang, J.; Liu, Y. Indoor acoustic localization: A survey. Hum. Centric Comput. Inf. Sci. 2020, 10, 2. [CrossRef]

38. Jang, B.; Kim, H. Indoor positioning technologies without offline fingerprinting map: A survey. IEEE Commun. Surv. Tutor. 2018, 21, 508-525. [CrossRef]

39. Vo, Q.D.; De, P. A survey of fingerprint-based outdoor localization. IEEE Commun. Surv. Tutor. 2015, 18, 491-506. [CrossRef]

40. Zhuang, Y.; Hua, L.; Qi, L.; Yang, J.; Cao, P.; Cao, Y.; Wu, Y.; Thompson, J.; Haas, H. A survey of positioning systems using visible LED lights. IEEE Commun. Surv. Tutor. 2018, 20, 1963-1988. [CrossRef]

41. Do, T.H.; Yoo, M. An in-depth survey of visible light communication based positioning systems. Sensors 2016, 16, 678. [CrossRef] [PubMed]

42. Ferreira, A.F.G.; Fernandes, D.M.A.; Catarino, A.P.; Monteiro, J.L. Localization and positioning systems for emergency responders: A survey. IEEE Commun. Surv. Tutor. 2017, 19, 2836-2870. [CrossRef]

43. Xiao, J.; Zhou, Z.; Yi, Y.; Ni, L.M. A survey on wireless indoor localization from the device perspective. ACM Comput. Surv. (CSUR) 2016, 49, 1-31. [CrossRef]

44. Davidson, P.; Piché, R. A survey of selected indoor positioning methods for smartphones. IEEE Commun. Surv. Tutor. 2016, 19, 1347-1370. [CrossRef]

45. Guo, G.; Chen, R.; Ye, F.; Peng, X.; Liu, Z.; Pan, Y. Indoor Smartphone Localization: A Hybrid WiFi RTT-RSS Ranging Approach. IEEE Access 2019, 7, 176767-176781. [CrossRef]

46. Jadaa, K.J.; Kamarudin, L.M.; Ahmad, R.B. Detection and Tracking Survey for Smart Home Using Wireless Sensor Network. J. Eng. Appl. Sci 2019, 14, 3119-3129.

47. Delaney, D.T.; Higgs, R.; O'Hare, G.M. A stable routing framework for tree-based routing structures in WSNs. IEEE Sens. J. 2014, 14, 3533-3547. [CrossRef]

48. Alaybeyoglu, A.; Kantarci, A.; Erciyes, K. A dynamic lookahead tree based tracking algorithm for wireless sensor networks using particle filtering technique. Comput. Electr. Eng. 2014, 40, 374-383. [CrossRef]

49. Mirsadeghi, M.; Mahani, A. Low power prediction mechanism for wsn-based object tracking. Procedia Technol. 2014, 17, 692-698. [CrossRef]

50. Gupta, A.; Patil, S.; Zaveri, M. Lost target recovery in wireless sensor network using tracking. In Proceedings of the 2012 International Conference on Communication Systems and Network Technologies, Rajkot, Gujarat, India, 11-13 May 2012; pp. 352-356.

51. Rouhani, S.; Haghighat, A.T. Boundary static clustering target tracking in wirless sensor networks. In Proceedings of the 2015 6th International Conference on Computing, Communication and Networking Technologies (ICCCNT), Dallas-Fortworth, TX, USA, 13-15 July 2015; pp. 1-6.

52. Wahdan, M.A.; Al-Mistarihi, M.F.; Shurman, M. Static cluster and dynamic cluster head (SCDCH) adaptive prediction-based algorithm for target tracking in wireless sensor networks. In Proceedings of the 2015 38th International Convention on Information and Communication Technology, Electronics and Microelectronics (MIPRO), Opatija, Croatia, 25-29 May 2015 ; pp. 596-600.

53. Zhou, M.; Zhang, J.J.; Papandreou-Suppappola, A. Multiple target tracking in urban environments. IEEE Trans. Signal Process. 2015, 64, 1270-1279. [CrossRef]

54. Amudha, J.; Arpita, P. Multi-Camera Activation Scheme for Target Tracking with Dynamic Active Camera Group and Virtual Grid-Based Target Recovery. Procedia Comput. Sci. 2015, 58, 241-248. [CrossRef]

55. Bhowmik, S.; Das, S.; Giri, C. Tree based tracking target in wireless sensor network. In Proceedings of the 2016 International Conference on Advances in Computing, Communications and Informatics (ICACCI), Jaipur, India, 21-24 September 2016; pp. 390-397. 
56. Jinan, R.; Raveendran, T. Particle filters for multiple target tracking. Procedia Technol. 2016, 24, 980-987. [CrossRef]

57. Darabkh, K.A.; Albtoush, W.Y.; Jafar, I.F. Improved clustering algorithms for target tracking in wireless sensor networks. J. Supercomput. 2017, 73, 1952-1977. [CrossRef]

58. Khakpour, S.; Pazzi, R.W.; El-Khatib, K. Using clustering for target tracking in vehicular ad hoc networks. Veh. Commun. 2017, 9, 83-96. [CrossRef]

59. Joshi, P.; Joshi, A. Prediction Based Moving Object Tracking in Wireless Sensor Network. Int. Res. J. Eng. Technol. 2017, 4, 3365-3368.

60. Xiao, K.; Wang, R.; Zhang, L.; Li, J.; Fun, T. ASMT: An augmented state-based multi-target tracking algorithm in wireless sensor networks. Int. J. Distrib. Sens. Netw. 2017, 13, 1550147717703115. [CrossRef]

61. Silva, A.; Zhou, F.; Pontes, E.; Simplicio, M.A.; Aguiar, R.; Guelfi, A.; Kofuji, S. Energy-efficient node position identification through payoff matrix and variability analysis. Telecommun. Syst. 2017, 65, 459-477. [CrossRef]

62. Oracevic, A.; Akbas, S.; Ozdemir, S. Secure and reliable object tracking in wireless sensor networks. Comput. Secur. 2017, 70, 307-318. [CrossRef]

63. Alshamaa, D.; Mourad-Chehade, F.; Honeine, P. Tracking of mobile sensors using belief functions in indoor wireless networks. IEEE Sens. J. 2017, 18, 310-319. [CrossRef]

64. Chen, Y.M.; Tsai, C.L.; Fang, R.W. TDOA/FDOA mobile target localization and tracking with adaptive extended Kalman filter. In Proceedings of the 2017 International Conference on Control, Artificial Intelligence, Robotics \& Optimization (ICCAIRO), Prague, Czech Republic, 20-22 May 2017; pp. 202-206.

65. Panag, T.S.; Dhillon, J. Dual head static clustering algorithm for wireless sensor networks. AEU Int. J. Electron. Commun. 2018, 88, 148-156. [CrossRef]

66. Zhang, H.; Zhou, X.; Wang, Z.; Yan, H.; Sun, J. Adaptive consensus-based distributed target tracking with dynamic cluster in sensor networks. IEEE Trans. Cybern. 2018, 49, 1580-1591. [CrossRef]

67. Qian, H.; Fu, P.; Li, B.; Liu, J.; Yuan, X. A novel loss recovery and tracking scheme for maneuvering target in hybrid WSNs. Sensors 2018, 18, 341. [CrossRef]

68. Zhang, C.; Fei, S. A Movement Algorithm for Target Group Tracking in Wireless Sensor Networks. In Proceedings of the 2018 37th Chinese Control Conference (CCC), Wuhan, China, 25-27 July 2018; pp. 7226-7230.

69. Li, W.; Han, C. A Novel Sensor Selection Algorithm for Multi-Target Tracking in Wireless Sensor Networks. In Proceedings of the 2018 Chinese Automation Congress (CAC), Xi'an, China, 30 November-2 December 2018; pp. 2854-2858.

70. Darabkh, K.A.; Alsaraireh, N.R. A yet efficient target tracking algorithm in wireless sensor networks. In Proceedings of the 2018 15th International Multi-Conference on Systems, Signals \& Devices (SSD), Yasmine Hammamet, Tunisia, 19-22 March 2018; pp. 7-11.

71. Liu, A.; Zhao, S. High-performance target tracking scheme with low prediction precision requirement in WSNs. Int. J. Ad Hoc Ubiquitous Comput. 2018, 29, 270-289. [CrossRef]

72. Luo, J.; Zhang, Z.; Liu, C.; Luo, H. Reliable and cooperative target tracking based on WSN and WiFi in indoor wireless networks. IEEE Access 2018, 6, 24846-24855. [CrossRef]

73. Yu, X.; Liang, J. Genetic fuzzy tree based node moving strategy of target tracking in multimodal wireless sensor network. IEEE Access 2018, 6, 25764-25772. [CrossRef]

74. Vilà-Valls, J.; Closas, P.; Bugallo, M.F.; Míguez, J. Distributed Multiple Gaussian Filtering for Multiple Target Localization in Wireless Sensor Networks. In Proceedings of the 2018 52nd Asilomar Conference on Signals, Systems, and Computers, Pacific Grove, CA, USA, 28-31 October 2018; pp. 1439-1443.

75. Izadi-Ghodousi, Z.; Hosseinpour, M.; Safaei, F.; Mohajerzadeh, A.H.; Alishahi, M. An Efficient Target Tracking in Directional Sensor Networks Using Adapted Unscented Kalman Filter. Wirel. Pers. Commun. 2019, 109, 1925-1954. [CrossRef]

76. Liang, C.; Wen, F.; Wang, Z. Trust-based distributed Kalman filtering for target tracking under malicious cyber attacks. Inf. Fusion 2019, 46, 44-50. [CrossRef]

77. Khan, M.A.; Khan, M.A.; Rahman, A.U.; Malik, A.W.; Khan, S.A. Exploiting cooperative sensing for accurate target tracking in industrial Internet of things. Int. J. Distrib. Sens. Netw. 2019, 15, 1550147719892203. [CrossRef]

78. Liu, E.; Chu, Y.; Zheng, L. Object Tracking Based on Compressive Features and Extreme Learning Machine. IEEE Access 2019, 7, 45994-46003. [CrossRef]

79. Nguyen, C.L.; Raza, U. LEMOn: Wireless Localization for IoT Employing a Location-Unaware Mobile Unit. IEEE Access 2019, 7 , 40488-40502. [CrossRef]

80. Ullah, I.; Liu, Y.; Su, X.; Kim, P. Efficient and accurate target localization in underwater environment. IEEE Access 2019, 7, 101415-101426. [CrossRef]

81. Belmonte-Hernández, A.; Hernández-Peñaloza, G.; Gutiérrez, D.M.; Álvarez, F. SWiBluX: Multi-Sensor Deep Learning Fingerprint for precise real-time indoor tracking. IEEE Sens. J. 2019, 19, 3473-3486. [CrossRef]

82. Liu, L.; Ji, H.; Zhang, W.; Liao, G. Multi-sensor multi-target tracking using probability hypothesis density filter. IEEE Access 2019, 7, 67745-67760. [CrossRef]

83. Liu, Q.; Lin, J.; Mao, Y. Adaptive Fuzzy Tree System for Target Tracking in Mission Critical Sensor Networks. IEEE Access 2019, 7, 184676-184685. [CrossRef] 
84. Mahmoudreza, H.; Hamid, K.; Mohammadtaghi, B. Augmented input estimation in multiple maneuvering target tracking. J. Syst. Eng. Electron. 2019, 30, 841-851.

85. Li, G.; Huang, Y.; Zhang, X.; Liu, C.; Shao, W.; Jiang, L.; Meng, J. Hybrid Maps Enhanced Localization System for Mobile Manipulator in Harsh Manufacturing Workshop. IEEE Access 2020, 8, 10782-10795. [CrossRef]

86. Fruhwirth-Reisinger, C.; Krispel, G.; Possegger, H.; Bischof, H. Towards Data-driven Multi-target Tracking for Autonomous Driving. In Proceedings of the 25th Computer Vision Winter Workshop (CVWW), Rogaska Slatina, Slovenia, 3-5 February 2020.

87. Li, P.; Yang, X.; Yin, Y.; Gao, S.; Niu, Q. Smartphone-Based Indoor Localization with Integrated Fingerprint Signal. IEEE Access 2020, 8, 33178-33187. [CrossRef]

88. Pérez-Solano, J.J.; Ezpeleta, S.; Claver, J.M. Indoor localization using time difference of arrival with UWB signals and unsynchronized devices. Ad Hoc Netw. 2020, 99, 102067. [CrossRef]

89. Wang, Y.; Yan, Y.; Li, Z.; Cheng, L. A Mobile Localization Method in Smart Indoor Environment Using Polynomial Fitting for Wireless Sensor Network. J. Sens. 2020, 2020. [CrossRef]

90. Alakhras, M.; Hussein, M.; Oussalah, M. Location fixing and fingerprint matching fingerprint map construction for indoor localization. J. Sens. 2020, 2020. [CrossRef]

91. Yucer, S.; Tektas, F.; Kilinc, M.V.; Kandemir, I.; Celebi, H.; Genc, Y.; Akgul, Y.S. RSSI-based Outdoor Localization with Single Unmanned Aerial Vehicle. arXiv 2020, arXiv:2004.10083.

92. Chen, Y.S.; Hsu, C.S.; Huang, C.Y.; Hung, H.M. Outdoor Localization for LoRaWans Using Semi-Supervised Transfer Learning with Grid Segmentation. In Proceedings of the 2019 IEEE VTS Asia Pacific Wireless Communications Symposium (APWCS), Singapore, 28-30 August 2019; pp. 1-5.

93. Haris, M.; Franzius, M.; Bauer-Wersing, U. Robust Outdoor Self-localization In Changing Environments. In Proceedings of the 2019 IEEE/RSJ International Conference on Intelligent Robots and Systems (IROS), Macau, China, 3-8 November 2019; pp. 714-719.

94. Kulshrestha, T.; Saxena, D.; Niyogi, R.; Cao, J. Real-time Crowd Monitoring using Seamless Indoor-Outdoor Localization. IEEE Trans. Mob. Comput. 2019, 19, 664-679. [CrossRef]

95. Sun, Y.; Shang, J.; Yang, Y. Indoor and Outdoor Seamless Localization Method Based on GNSS and WLAN. In International Conference in Communications, Signal Processing, and Systems; Springer: Singapore, 2018; pp. 1412-1419.

96. Kulshrestha, T.; Saxena, D.; Niyogi, R.; Raychoudhury, V.; Misra, M. SmartITS: Smartphone-based identification and tracking using seamless indoor-outdoor localization. J. Netw. Comput. Appl. 2017, 98, 97-113. [CrossRef]

97. Li, H.; He, Y.; Cheng, X.; Zhu, H.; Sun, L. Security and privacy in localization for underwater sensor networks. IEEE Commun. Mag. 2015, 53, 56-62. [CrossRef]

98. Tiku, S.; Pasricha, S. Overcoming Security Vulnerabilities in Deep Learning-based Indoor Localization Frameworks on Mobile Devices. ACM Trans. Embed. Comput. Syst. (TECS) 2019, 18, 1-24. [CrossRef]

99. Xue, Y.; Su, W.; Wang, H.; Yang, D.; Jiang, Y. DeepTAL: Deep Learning for TDOA-Based Asynchronous Localization Security with Measurement Error and Missing Data. IEEE Access 2019, 7, 122492-122502. [CrossRef]

100. Liu, Y.; Zhu, X.; Zhao, X.; Cao, Y. Adversarial learning for constrained image splicing detection and localization based on atrous convolution. IEEE Trans. Inf. Forensics Secur. 2019, 14, 2551-2566. [CrossRef]

101. Shen, L.; Ma, J.; Liu, X.; Wei, F.; Miao, M. A Secure and Efficient ID-Based Aggregate Signature Scheme for Wireless Sensor Networks. IEEE Internet Things J. 2017, 4, 546-554. [CrossRef]

102. Saleem, A.; Khan, A.; Malik, S.U.R.; Pervaiz, H.; Malik, H.; Alam, M.; Jindal, A. FESDA: Fog-Enabled Secure Data Aggregation in Smart Grid IoT Network. IEEE Internet Things J. 2019, 7, 6132-6142. [CrossRef]

103. Qureshi, U.M.; Umair, Z.; Hancke, G.P. Evaluating the implications of varying Bluetooth low energy (BLE) transmission power levels on wireless indoor localization accuracy and precision. Sensors 2019, 19, 3282. [CrossRef]

104. Saboor, A.; Ahmad, R.; Ahmed, W.; Kiani, A.K.; Alam, M.M.; Kuusik, A.; Le Moullec, Y. Dynamic Slot Allocation Using Non Overlapping Backoff Algorithm in IEEE 802.15.6 WBAN. IEEE Sens. J. 2020, 20, 10862-10875. [CrossRef]

105. Yuan, G.; Ze, Z.; Changcheng, H.; Chuanqi, H.; Li, C. In-vehicle localization based on multi-channel Bluetooth Low Energy received signal strength indicator. Int. J. Distrib. Sens. Netw. 2020, 16, 1550147719900093. [CrossRef]

106. Yang, M.; Wang, A.; Sun, G.; Zhang, Y. Deploying charging nodes in wireless rechargeable sensor networks based on improved firefly algorithm. Comput. Electr. Eng. 2018, 72, 719-731. [CrossRef]

107. Saboor, A.; Ahmad, R.; Ahmed, W.; Kiani, A.K.; Moullec, Y.L.; Alam, M.M. On Research Challenges in Hybrid Medium-Access Control Protocols for IEEE 802.15.6 WBANs. IEEE Sens. J. 2019, 19, 8543-8555. [CrossRef]

108. Saeed, N.; Celik, A.; Al-Naffouri, T.Y.; Alouini, M.S. Localization of energy harvesting empowered underwater optical wireless sensor networks. IEEE Trans. Wirel. Commun. 2019, 18, 2652-2663. [CrossRef]

109. Del Prete, M.; Decarli, N.; Masotti, D.; Dardari, D.; Costanzo, A. Exploitation of multi-sine intermodulation for passive backscattering UWB localization. In Proceedings of the 2018 IEEE/MTT-S International Microwave Symposium-IMS, Philadelphia, PA, USA, 10-15 June 2018; pp. 262-265.

110. Jo, S.H.; Yoon, H.; Shin, Y.C.; Choi, W.; Park, C.S.; Kim, M.; Youn, B.D. Designing a phononic crystal with a defect for energy localization and harvesting: Supercell size and defect location. Int. J. Mech. Sci. 2020, 179, 105670. [CrossRef] 
111. Shearwood, J.; Hung, D.M.Y.; Cross, P.; Preston, S.; Palego, C. Honey-bee localization using an energy harvesting device and power based angle of arrival estimation. In Proceedings of the 2018 IEEE/MTT-S International Microwave Symposium-IMS, Philadelphia, PA, USA, 10-15 June 2018; pp. 957-960.

112. Sharma, A.; Kakkar, A. Forecasting daily global solar irradiance generation using machine learning. Renew. Sustain. Energy Rev. 2018, 82, 2254-2269. [CrossRef]

113. Chen, F.; Fu, Z.; Yang, Z. Wind power generation fault diagnosis based on deep learning model in internet of things (IoT) with clusters. Clust. Comput. 2019, 22, 14013-14025. [CrossRef]

114. Zhu, Y.; Yan, F.; Zhang, Y.; Zhang, R.; Shen, L. SDN-Based Anchor Scheduling Scheme for Localization in Heterogeneous WSNs. IEEE Commun. Lett. 2017, 21, 1127-1130. [CrossRef]

115. Nabati, M.; Navidan, H.; Shahbazian, R.; Ghorashi, S.A.; Windridge, D. Using Synthetic Data to Enhance the Accuracy of Fingerprint-Based Localization: A Deep Learning Approach. IEEE Sens. Lett. 2020, 4, 1-4. [CrossRef]

116. Singh, S.; Kumar, K.; Gupta, S. Machine Learning based Indoor Localization Techniques for Wireless Sensor Networks. In Proceedings of the 2020 2nd International Conference on Advances in Computing, Communication Control and Networking (ICACCCN), Greater Noida, India, 18-19 December 2020; pp. 373-380.

117. Akhil, K.; Sinha, S. Self-Localization in Large Scale Wireless Sensor Network Using Machine Learning. In Proceedings of the 2020 International Conference on Emerging Trends in Information Technology and Engineering (ic-ETITE), Vellore, India, 24-25 February 2020; pp. 1-5.

118. Konecny, J.; Prauzek, M.; Martinek, R.; Michalek, L.; Tomis, M. Real-time Patient Localization in Urgent Care: System Design and Hardware Perspective. In Proceedings of the 2018 IEEE 20th International Conference on e-Health Networking, Applications and Services (Healthcom), Ostrava, Czech Republic, 17-20 September 2018; pp. 1-5.

119. Wan, L.; Han, G.; Shu, L.; Feng, N. The critical patients localization algorithm using sparse representation for mixed signals in emergency healthcare system. IEEE Syst. J. 2015, 12, 52-63. [CrossRef]

120. Sierotowicz, M.; Connan, M.; Castellini, C. Human-In-The-Loop Assessment of an Ultralight, Low-Cost Body Posture Tracking Device. Sensors 2020, 20, 890. [CrossRef] [PubMed]

121. Quan, W.; Woo, J.; Toda, Y.; Kubota, N. Human Posture Recognition for Estimation of Human Body Condition. J. Adv. Comput. Intell. Intell. Inform. 2019, 23, 519-527. [CrossRef]

122. Arafat, M.Y.; Moh, S. Localization and clustering based on swarm intelligence in UAV networks for emergency communications. IEEE Internet Things J. 2019, 6, 8958-8976. [CrossRef]

123. Saboor, A.; Ahmad, R.; Ahmed, W.; Alam, M.M. A Unique Backoff Algorithm in IEEE 802.15.6 WBAN. In Proceedings of the 2018 IEEE 88th Vehicular Technology Conference (VTC-Fall), Chicago, IL, USA, 27-30 August 2018; pp. 1-5. [CrossRef]

124. Cheng, L.; Li, Y.; Xue, M.; Wang, Y. An Indoor Localization Algorithm based on Modified Joint Probabilistic Data Association for Wireless Sensor Network. IEEE Trans. Ind. Inform. 2020, 17, 63-72. [CrossRef]

125. Dang, X.; Cheng, Q.; Zhu, H. Indoor Multiple Sound Source Localization via Multi-Dimensional Assignment Data Association. IEEE/ACM Trans. Audio Speech Lang. Process. 2019, 27, 1944-1956. [CrossRef]

126. Ge, T.; Tharmarasa, R.; Lebel, B.; Florea, M.; Kirubarajan, T. Target Localization and Sensor Synchronization in the Presence of Data Association Uncertainty. In Proceedings of the 2019 22th International Conference on Information Fusion (FUSION), Ottawa, ON, Canada, 2-5 July 2019; pp. 1-6. 